\title{
Long-range road geometry estimation using moving vehicles and road-side observations
}

\author{
Lars Hammarstrand, Maryam Fatemi, Ángel F. García-Fernández, and Lennart Svensson
}

\begin{abstract}
This paper presents an algorithm for estimating the shape of the road ahead of a host vehicle equipped with the following onboard sensors: a camera, a radar and vehicle internal sensors. The aim is to accurately describe the road geometry up to $200 \mathrm{~m}$ ahead in highway scenarios. This purpose is accomplished by deriving a precise clothoid-based road model for which we design a Bayesian fusion framework. Using this framework the road geometry is estimated using sensor observations on the shape of the lane markings, the heading of leading vehicles and the position of road side radar reflectors. The evaluation on sensor data shows that the proposed algorithm is capable of capturing the shape of the road well, even in challenging mountainous highways.
\end{abstract}

Index Terms-Road geometry, Bayesian Estimation, Advanced driver assistance systems.

\section{INTRODUCTION}

Advanced driver assistance systems (ADAS) aim at providing drivers with a safer and more convenient driving experience. These systems monitor the current traffic situation and support the driver in dangerous situations by generating a warning, or by autonomously intervening in order to prevent an accident or to mitigate its consequences. It is crucial for ADAS systems to make informed decisions in a timely manner since they are designed to act prior to an accident. As such, some of these systems need knowledge of the shape of the road to be able to make a decision. Among other things, road geometry helps the system to answer important questions, e.g. 'Is the vehicle in front in my lane?".

Estimates of the road geometry are typically based on observations from different sensors as for instance camera, radar and lidar. In the case of vision-based systems, the road geometry is estimated by detecting visual cues like lane markings and road edges. A survey of such methods can be found in [1] and [2]. Although this type of system can give accurate estimates close to the host vehicle (approx. up to 60 $\mathrm{m}$ ) in many situations, issues such as worn-out lane markings, adverse weather or ambient light, can cause serious difficulties. Additionally, these systems suffer from poor effective resolution of the lane markings at far distances [3]. Radar-based systems, on the other hand, can sense objects up to longer distances and are less sensitive to both weather and ambient light. The radar-only systems typically estimate the road using

Lars Hammarstrand, Maryam Fatemi and Lennart Svensson are with the Department of Signals and Systems, Chalmers University of Technology, SE-412 96 Gothenburg, Sweden (emails: \{lars.hammarstrand, maryam.fatemi, lennart.svensson \}@ chalmers.se).

Ángel F. García-Fernández is with the Department of Electrical and Computer Engineering, Curtin University, Perth, WA 6102, Australia (email angel.garciafernandez@curtin.edu.au) observations of stationary objects by the road. Examples of such systems are presented in [4]-[6]. For lidar based systems, an overview can be found in [7] which discusses both online and offline methods for road extraction from lidar data. The main strength with lidar based systems is that they are able to detect lane markings [8] as well as road boundaries [9], [10]. Its drawbacks are, similar to vision based systems, weather sensitivity and limited detection range (typically $60 \mathrm{~m}$ ).

Clearly, radar and vision based systems provide complementary information and fusing the two can lead to increased accuracy and robustness. For example, in [11], lane marking measurements from a camera are fused with measurements of road boundaries from a radar sensor. In other works, radar observations of leading vehicles are incorporated into the fusion system to improve performance [12]-[16]. In [17], we propose a fusion algorithm that, in addition the above, takes stationary road side radar observations in to account.

The above mentioned online methods, except [17], focus on estimating the near-range road geometry, approximately up to $60 \mathrm{~m}$. The focus on near-range stems from two limiting factors, camera and lidar sensors do not give observations at farther distances and/or limited flexibility in the road model used. In this paper, we estimate the far-range road geometry, up to $200 \mathrm{~m}$ ahead of the host vehicle, in highway scenarios by using observations of lane markings coming from a camera sensor and observations leading vehicles and road side objects from a radar-camera fusion system. Furthermore, we propose a flexible clothoid-based segmented road model, where the segments are connected in a manner that ensures $G^{2}$-continuity along the curve. In addition to estimating the road geometry we also estimate road-side barriers. Besides simplifying the inference, knowledge of the road barriers can be useful in subsequent threat assessment algorithms as the barriers / boundaries indicate the edges of a driving corridor.

The differences of this paper compared to [17] are in the proposed road model, how we treat the stationary road-side radar measurements and more extended evaluations. More specifically, the proposed clothoid-based road model in this paper allows for calculating continuous curvature and heading along the road. The road in [17] was modeled by discrete points, therefore, we could only directly access the curvature at discrete points along the road. Moreover, in [17] the guard rail measurements are preprocessed and we make hard decisions regarding which measurements are generated by the guard rails and which are clutter. Based on data analysis, where we identify the guard rail posts as strong radar reflectors, we instead form a probabilistic model of the guard rail observations. Using this model in a probabilistic multiple 
hypothesis framework we can avoid discarding measurements by making hard decisions and, hence, more efficiently use the available information. We evaluate our algorithm using real data recorded in different types of highways, i.e., straight and winding highways as well as highways belonging to the curvier end of the spectrum.

\section{PROBLEM FORMULATION AND SYSTEM DESCRIPTION}

This paper considers the problem of online estimation of the shape of the road, i.e., the geometry of the road, up to $200 \mathrm{~m}$ ahead of a host vehicle in highway scenarios using onboard sensors. More specifically, we consider a vehicle fitted with a forward-looking dual-mode radar, a forward-looking camera and internal vehicle sensors such as wheel speed sensors, gyroscopes and accelerometers. While the internal sensors measure the movement of the host vehicle, the camera is capable of tracking the lane markings as well as detecting and classifying leading vehicles. The radar observes the position and velocity of other vehicles as well as stationary road-side objects, e.g. guard rail posts. The information about leading vehicles is pre-processed by the radar-camera system to give fused estimates of their velocity and position.

\section{A. Assumptions and limitations}

As this paper focuses on highways and a given sensor setup, to make the problem more concise we introduce a set of simplifying assumptions. Firstly, the scope is limited to estimating the shape of a single road, i.e., intersections, exits and forks are not described by the system until the host vehicle has actually left the current road. Many interesting scenarios are not dependent on handling multiple roads, however; this limitation can cause some confusion as, e.g., a leading vehicle taking an exit.

Secondly, as we do not considering forks or exits, our proposed methods can not readily be applied to other types of roads, e.g., rural or inner-city roads. In addition, we make other assumptions about the behavior of other vehicles and stationary radar detections that are not valid in these scenarios. For these types of roads and at these short distances, it is probably quite sufficient to mainly rely on lane marking measurement from the camera system as studied in [1], [2].

Thirdly, we assume that the road geometry can be described in a flat 2D plane, i.e., no slope information is considered. This is a common assumption applied in much of the road geometry estimation literature, see [18]-[21]. The main reason that we do not include it here, however, is that slope information is not present in the sensor observations available. Having access to such observations via detailed maps, 3D laser scanners or stereo camera systems would clearly motivate introducing the third dimension in the geometry model. Not considering slope information limits the estimation accuracy in hilly roads.

Lastly, as is common in this field, the sensors pre-process (filter) their measurements. However, as we do not receive any error covariance nor have insight into these filters we are limited to treat the observations as measurements instead of estimates in our fusion system. We further assume that there exists a fusion system like the one described in [22] and

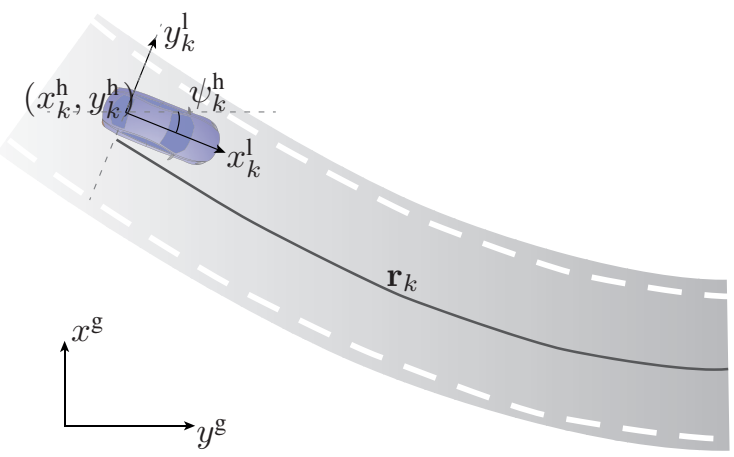

Figure 1. Two Cartesian coordinate systems, one fixed global, $\left(x^{\mathrm{g}}, y^{\mathrm{g}}\right)$, and one local moving coordinate frame attached to the host vehicle, $\left(x_{k}^{1}, y_{k}^{1}\right)$.

used in, e.g., [6], [23], [24] capable of handling asynchronous sensor data and estimating the host vehicle state. Moreover, as the the estimate of the host vehicle state (velocity and acceleration) from this system has negligible uncertainty in comparison to the uncertainty in the road geometry [25] we regard the host vehicle state as a control input to our models.

\section{B. Notation and road geometry definition}

In order to estimate the road geometry we first need to clearly define it. As in [17], we use the following definition of the road geometry:

\section{The geometry of the road is defined as the shape of}

the middle of the host vehicle's lane.

That is, for a given time instance $k$, we assume that there exists a mathematical expression for the current shape (geometry) of the middle of the host vehicle's lane. Further, the geometry description is parameterized using a time varying road state vector, $\mathbf{r}_{k}$, which is defined in Section III-A.

The geometry is expressed in a local Cartesian coordinate system, $\left(x_{k}^{l}, y_{k}^{l}\right)$, attached to the middle of the rear axle of the host vehicle with its $x$-axis pointing in the direction of travel, as depicted in Figure 1. Note that this local coordinate frame is fixed to the host vehicle and thus moves with it. The pose of this local coordinate frame is expressed in a stationary global Cartesian coordinate system as $\left(x_{k}^{\mathrm{h}}, y_{k}^{\mathrm{h}}\right)$ and $\psi_{k}^{\mathrm{h}}$. The time index $k$ will be used throughout this paper to refer to a time instance $t_{k}$ for which the $k$ th measurement in total (from any of the sensors) was taken. As the sensors are asynchronous, the time between two measurements $T_{k}=t_{k}-t_{k-1}$ is not constant.

\section{Road observations}

The onboard sensors deliver three different types of observations on the road geometry. The camera sensor detects and estimates the shape of the lane markings, both the radar and the camera detect moving vehicles and the radar gives us stationary detections from road-side objects. These observations are described in more detail below.

1) Lane marking observations: If present and detected by the camera system, the shapes of the left and right lane markings closest to the host vehicle are described using third 
degree polynomials. The polynomials are given in the local vehicle coordinate frame $\left(x_{k}^{1}, y_{k}^{1}\right)$ as,

$$
\begin{aligned}
& y_{k}^{1}=l_{k}^{0}+l_{k}^{1} x_{k}^{1}+l_{k}^{2}\left(x_{k}^{1}\right)^{2}+l_{k}^{3}\left(x_{k}^{1}\right)^{3} \\
& y_{k}^{\mathrm{r}}=r_{k}^{0}+r_{k}^{1} x_{k}^{1}+r_{k}^{2}\left(x_{k}^{1}\right)^{2}+r_{k}^{3}\left(x_{k}^{1}\right)^{3}
\end{aligned}
$$

for the left lane marking and the right lane marking, respectively. Roughly every $100 \mathrm{~ms}$, the camera system delivers the polynomial coefficients for each side, $\mathbf{z}_{k}^{1}=\left[l_{k}^{0}, l_{k}^{1}, l_{k}^{2}, l_{k}^{3}\right]^{T}$ and $\mathbf{z}_{k}^{\mathrm{r}}=\left[r_{k}^{0}, r_{k}^{1}, r_{k}^{2}, r_{k}^{3}\right]^{T}$, independently of each other. Additionally, each coefficient vector is coupled with a confidence value (between 0 and 3 ) and an indication of the maximum distance for which the polynomials (1) - (2) are valid, denoted $x_{\max }^{1}$ and $x_{\max }^{\mathrm{r}}$, respectively.

2) Object observations: The radar-camera fusion system delivers object estimates every $25 \mathrm{~ms}$. Based on their speed over-ground and camera classification, these estimates are divided into two categories, moving/stationary vehicles or stationary unclassified objects. The latter category typically includes stationary road-side objects reflecting radar energy, such as guard-rail posts. We denote the vehicle estimates as $\mathbf{z}_{k}^{\mathrm{v}}$ and the stationary detections as $\mathbf{z}_{k}^{\mathrm{s}}$.

For the $i^{t h}$ detected vehicle, the fusion system provides the following description,

$$
\mathbf{z}_{k}^{\mathrm{v}, i}=\left[x_{k}^{i}, y_{k}^{i}, \phi_{k}^{i}, v_{k}^{i}\right]^{T}
$$

where $\left[x_{k}^{i}, y_{k}^{i}\right]$ is the position of the vehicle in the local coordinate frame, $\phi_{k}^{i}$ is its heading direction and $v_{k}^{i}$ is its speed over-ground.

For the stationary objects the description is simply given as the position in the local coordinate frame.

\section{Estimation problem}

The aim is to estimate the geometry of the road (including uncertainty) using the available observations from the onboard sensors. More specifically, we want to recursively calculate the posterior density of the road state, $p\left(\mathbf{r}_{k} \mid \mathbf{z}_{1: k}\right)$, where $\mathbf{z}_{1: k}$ is the set of all observations up to the current time index $k$.

\section{ROAD MODEL}

We define and motivate our proposed model of the road in Section III-A, derive approximations to make the model suitable in a filtering framework in Section III-B and finally compare our approximation with the commonly used thirddegree polynomial model in Section III-C.

\section{A. Exact road model}

Roads are typically built in a manner which ensures a smooth ride for the road users. Among other things, there should be no abrupt changes in the road curvature. To accommodate this, it is common to construct roads where straight sections are connected with constant curvature segments using a transition curve, i.e., a curve with linearly changing curvature [26]. Our proposed model is based on this notion.
In the most general form, the $2 \mathrm{D}$ geometry of the road can be described using a 2-dimensional parametric curve $\mathbf{c}_{r}(s)$ expressed in arc length $s$ (distance along the road), as

$$
\mathbf{c}_{r}(s)=\left[\begin{array}{l}
x_{r}(s) \\
y_{r}(s)
\end{array}\right]
$$

where $\left(x_{r}(s), y_{r}(s)\right)$ is the position of the road at arc length $s$ in the local coordinate system. The parameters used to describe $\mathbf{c}_{r}(\cdot)$ are collected in the road state $\mathbf{r}_{k}$. That is, if we know $\mathbf{r}_{k}$ we also know the shape of the road. Below, we will introduce how we propose to model (4) and consequently how we choose to parameterize the road state.

We propose to model the road using connected segments fixed to the road. Fixing the model to the road rather than to the vehicle, which is a common modeling choice, allows more efficient use of our observations. As the road model in large parts is static rather than dynamically changing with the movement of the host vehicle, there is no need to add process noise in order to account for changes in the road.

Each segment in our model is described using a parametric curve (clothoid) of length $L$ that has a linearly changing curvature as a function of $s$. The curvature of the $i$ th-segment, denoted $\kappa_{r}^{i}(s)$, is thus expressed as

$$
\kappa_{r}^{i}(s)=\kappa_{0}^{i}+\kappa_{1}^{i} s,
$$

where $\kappa_{0}^{i}$ is the initial curvature of the segment and $\kappa_{1}^{i}$ is its curvature change rate. As a consequence of (5), the heading of the road in each segment, $\varphi_{r}^{i}(s)$, is described as

$$
\varphi_{r}^{i}(s)=\varphi_{0}^{i}+\kappa_{0}^{i} s+\frac{\kappa_{1}^{i}}{2} s^{2}
$$

where $\varphi_{0}^{i}$ is the initial heading of the road at the start of the $i$ th segment. From (6) we can finally describe the geometry of the $i$ th segment using the following integral

$$
\left[\begin{array}{l}
x_{r}^{i}(s) \\
y_{r}^{i}(s)
\end{array}\right]=\left[\begin{array}{c}
x_{0}^{i} \\
y_{0}^{i}
\end{array}\right]+\int_{0}^{s}\left[\begin{array}{c}
\cos \left(\varphi_{r}^{i}(t)\right) \\
\sin \left(\varphi_{r}^{i}(t)\right)
\end{array}\right] d t
$$

where $\left(x_{0}^{i}, y_{0}^{i}\right)$ are the initial coordinates of the $i$ th segment.

Using (7) we can describe the geometry of each segment in the road model. In order for the segments to align smoothly, the segments should be connected such that the complete road has $G^{2}$-continuity (2nd order geometric), i.e., the position, heading and curvature of two connected segments should be equal at the intersection. This is ensured by introducing the following constraints on the initial conditions,

$$
\begin{aligned}
\kappa_{0}^{i} & =\kappa_{0}^{i-1}+\kappa_{1}^{i-1} L \\
\varphi_{0}^{i} & =\varphi_{0}^{i-1}+\kappa_{0}^{i-1} L+\kappa_{1}^{i-1} L^{2} / 2 \\
x_{0}^{i} & =x_{r}^{i-1}(L) \\
y_{0}^{i} & =y_{r}^{i-1}(L)
\end{aligned}
$$

Note that, as the initial curvature, heading and position are fixed, the only free parameter for each segment is the constant curvature change rate.

As we are only interested in the describing the road 200 meters ahead of the host vehicle it is sufficient that we consider the $N$ closest segments such that $N L \geq 200$. Let us denote the indices of these segments as $i_{1}, i_{2} \ldots, i_{N}$ and 


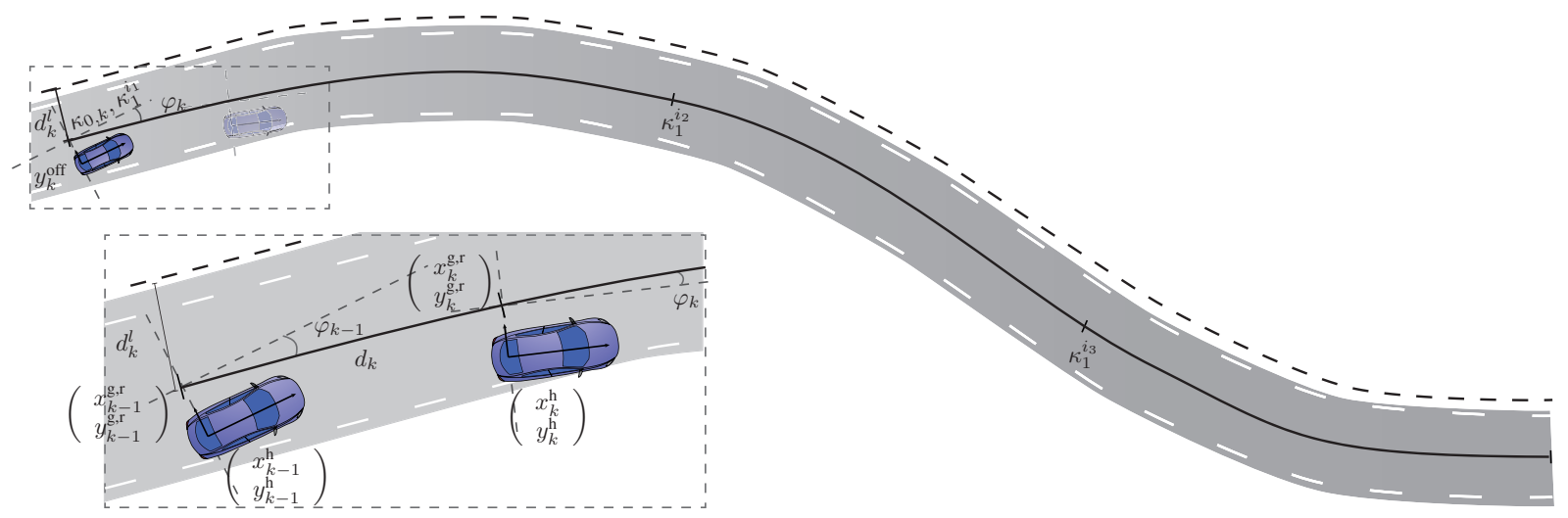

Figure 2. Illustration of the road model, state parametrization and process model. The road is characterized by initial offset $y_{k}^{\text {off }}$, initial heading $\varphi_{k}$, initial curvature $\kappa_{0, k}$ and curvature change rate of each segment $\kappa_{1}^{i_{1}}, \ldots, \kappa_{1}^{i_{N}}$. The left barrier is assumed to be parallel to the road and characterized by the initial offset $d_{k}^{l}$.

the corresponding curvature change rates as $\kappa_{1}^{i_{1}}, \kappa_{1}^{i_{2}}, \ldots, \kappa_{1}^{i_{N}}$. Note that, as the curvature change rates describe a fixed part of the road and are assumed constant for each segment, the length of each segment, $L$, needs to be chosen such that the model is flexible enough to capture changes in the curvature change rate. Furthermore, as we have no prior knowledge regarding where the curvature change rate changes so we need to account for this as well. For the scenarios covered in this paper we have chosen $L=50 \mathrm{~m}$ and $N=5$.

Further, the geometry of the road is expressed in the local coordinate frame and, thus, it changes as the car moves. To relate the road to the position of the host vehicle we require the initial position $\left(0, y_{k}^{\text {off }}\right)$, heading $\varphi_{k}$ and curvature $\kappa_{0, k}$ at the current position of the car. Note that, by setting the initial position to $\left(0, y_{k}^{\text {off }}\right)$ we assume that the current representation of the road starts at the rear axle of the host vehicle (local coordinate frame). The road state also includes the width of the host lane and is defined as:

$$
\mathbf{r}_{k}=\left[y_{k}^{\text {off }}, \varphi_{k}, \kappa_{0, k}, \kappa_{1}^{i_{1}}, \ldots, \kappa_{1}^{i_{n}}, w_{k}^{\text {lane }}\right]^{T} .
$$

In order to preserve the description of the geometry when the host performs a lane change (the road geometry gets shifted a lane width), it is convenient to have dynamic segment lengths denoted, $\ell_{k}^{i}$, for $i=i_{1} \ldots i_{N}$. The dynamic segment lengths are compensated both for the movement of the host ${ }^{1}$ as well as if the host makes a lane change. Nevertheless, as we assume that the dynamics of the host vehicle is known, this quantity is known and does not need to be included in the state vector More details is given in Section IV.

\section{B. Approximative road model}

In order to use this road model in a filter it is convenient to have a closed-form solution to (7). However, the integral in (7) does not have an analytical solution. Thus, we propose to approximate the trigonometric functions in (7) using third-order Taylor series expansion around $s=L / 2$. Although there exist more accurate approximations of (7) [27] - [28], numerical

\footnotetext{
${ }^{1}$ For example, if the vehicle as moved $d \mathrm{~m}$ since last time, the first segmen needs to be shortened $d \mathrm{~m}$.
}

comparison shows that a third-order Taylor expansion is less computationally complex and yields sufficient accuracy for relevant road shapes, see Figure 3. That is, the limiting factor regarding segment length is not the numerical accuracy of the approximation but rather how long the constant curvature change rate assumption is accurate.

Let $\tilde{x}_{r}^{i}(s)$ and $\tilde{y}_{r}^{i}(s)$ be the approximated Cartesian positions of the $i$ th segment resulting from the Taylor expansion, which is detailed in Appendix A. With these approximations, the parametric curve can be described as

$$
\tilde{\mathbf{c}}_{r}(s)=\sum_{j=1}^{N}\left[\begin{array}{c}
\tilde{x}_{r}^{i_{j}}\left(\Delta s^{i}\right) \\
\tilde{y}_{r}^{i_{j}}\left(\Delta s^{i}\right)
\end{array}\right] \chi_{A_{i}}(s)
$$

where $\chi_{A_{i}}(s)$ is an indicator function which is one if $s$ is in the intervall $A_{i}=\left[\sum_{n=1}^{i-1} \ell_{k}^{n}, \sum_{n=1}^{i} \ell_{k}^{n}\right]$ and $\Delta s^{i}=s-\sum_{n=1}^{i-1} \ell_{k}^{n}$. Similarly, the heading, $\varphi_{r}(s)$, and curvature, $\kappa_{r}(s)$, of the road are given by

$$
\begin{aligned}
& \varphi_{r}(s)=\sum_{j=1}^{N} \varphi_{r}^{i}\left(\Delta s^{i}\right) \chi_{A_{i}}(s) \\
& \kappa_{r}(s)=\sum_{j=1}^{N} \kappa_{r}^{i}\left(\Delta s^{i}\right) \chi_{A_{i}}(s)
\end{aligned}
$$

where $\varphi_{r}^{i}(\cdot)$ is given by (6) and $\kappa_{r}^{i}(\cdot)$ is given by (5).

In addition, to be able to relate the stationary road side observations from the radar to the shape of the road, we propose to include a barrier model in our state representation. We assume that the road barrier can be modeled as parallel to the middle of the host lane with a constant offset over the observable region. That is, we introduce a barrier state vector

$$
\mathbf{b}_{k}=\left[e_{k}^{1}, d_{k}^{\mathrm{l}}, e_{k}^{\mathrm{r}}, d_{k}^{\mathrm{r}}\right]^{T}
$$

where $d_{k}^{1}$ and $d_{k}^{\mathrm{r}}$ are the lateral distance from the middle of the host lane to left and right barrier, respectively. In contrast to the road, the left and right barrier are not always present. To treat the existence / nonexistence of the barriers we introduce the indicator variables $e_{k}^{1} \in\{0,1\}$ and $e_{k}^{\mathrm{r}} \in\{0,1\}$, which if set to 1 indicates the existence of the respective barrier. 


\section{Comparison to the linear cubic polynomial model}

A commonly used geometry model in the lane tracking literature is to describe the shape of the lane markings using a cubic polynomial [29]

$$
\left[\begin{array}{l}
x_{r}(x) \\
y_{r}(x)
\end{array}\right]=\left[\begin{array}{l}
x_{0} \\
y_{0}
\end{array}\right]+\left[\begin{array}{c}
x \\
\phi_{0} x+\frac{c_{0}}{2} x^{2}+\frac{c_{1}}{6} x^{3}
\end{array}\right]
$$

where $x_{r}$ and $y_{r}$ are the $x$ - and $y$-coordinates of the road in the local coordinate frame, and $\phi_{0} \approx \varphi_{0}, c_{0} \approx \kappa_{0}$ and $c_{1} \approx \kappa_{1}$. The approximation is basically performed using the small angle approximation for trigonometric functions (first order Taylor expansion around $s=0$ ) on (7) and approximating the arc length as the $x$-component. For a full derivation of this approximation, see [29], [30]. The main advantages with this model is that its fairly accurate when describing roads up to 60 $\mathrm{m}$ [31] and when the change in heading angle is smaller than about 15 degrees [30]. Furthermore, as the model is linear in its parameters which allows for simple estimation. However, as investigated in [32] the polynomial model is unsuitable in a fusion framework as small errors in the higher oder parameters can lead to large errors in the low order parameters.

Less investigated, however, is the use of this model to describe segments in a segmented road model and the physical interpretability of its model parameters. As such, we proceed to compare the accuracy of the model given by (17) with our proposed road model (13) in these aspects. In the comparison we use a reference clothoid road model (7) having two segment with $L=100 \mathrm{~m}, y_{0}=x_{0}=0, \varphi_{0}=0$. The parameters $\kappa_{0}$, $\kappa_{1}^{1}$ and $\kappa_{1}^{2}$ are set to follow zero mean Gaussian distributions describing curvy highways with respective standard deviations of $1 / 750 \mathrm{~m}^{-1}$ in curvature and $5 \cdot 10^{-5} \mathrm{~m}^{-2}$ in curvature change rate.

The accuracy of the two approximative models is evaluated using Monte Carlo simulations where we draw the parameters describing the true clothoid from the parameter distribution above. To get a comparison of the physical interpretability of the models, the same parameter values are used in both the approximative models as for the true clotoid. For simulation $i$ with road state $\mathbf{r}_{i}$, the modeling error of (13) is calculated as

$$
\epsilon_{i}(s)=\left\|\tilde{\mathbf{c}}_{r_{i}}(s)-\mathbf{c}_{r_{i}}(s)\right\|_{2}
$$

and similarly for (17). The mean error over the simulations of each model is depicted in Figure 3 together with the error variance. It is clear from the results that the cubic polynomial model suffers from propagating modeling errors and is not able to accurately describe the road up to $200 \mathrm{~m}$ ahead, while the more accurate model approximation is able to keep the error bounded to at most around $1 \mathrm{~cm}$. It is also clear that the physical interpretability of the parameters in the polynomial model is limited in this scenario.

\section{PROCESS MODEL}

The describe the process model for the road state, we divided it into two parts. The first part describes the road in relation to the host vehicle (first three states), whereas the second part describes the actual shape of the current road segments. The process model for the former is presented in
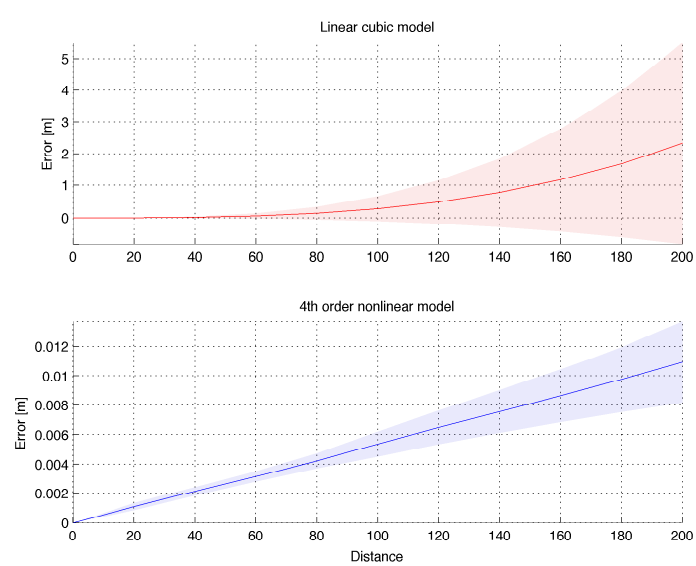

Figure 3. Monte Carlo comparison between two 2-segment road models having segment lengths $100 \mathrm{~m}$, using the linear cubic model (above) and a 4th order nonlinear model (below). Both are evaluated against a reference clothoid having the same length and parameters. The mean error (Euclidian distance at arc-length $s$ ) and error variance is calculated for roads having parameters drawn from the following parameter densities, $\varphi_{0}=0, \kappa_{0} \sim$ $\mathcal{N}\left(0,(1 / 750)^{2}\right)$ and $\kappa_{1} \sim \mathcal{N}\left(0,\left(5 \cdot 10^{-5}\right)^{2}\right)$.

Section IV-A and the latter in Section IV-B. Additionally, we describe how to compensate the road state in the event that the host makes a lane change in Section IV-C.

\section{A. Host fixed process model}

The first three elements of our state vector ( $y_{k}^{\text {off }}, \varphi_{k}$ and $\kappa_{0, k}$ ), describe the road relative to the current pose of the host vehicle. To describe their process model we, thus, need to compensate for the host movement.

The position and orientation of the host vehicle in a fixed global coordinate system are received from the fusion system. These parameters, at previous and current time stamps, are denoted by $\left(x_{k-1}^{\mathrm{h}}, y_{k-1}^{\mathrm{h}}, \psi_{k-1}^{\mathrm{h}}\right)$ and $\left(x_{k}^{\mathrm{h}}, y_{k}^{\mathrm{h}}, \psi_{k}^{\mathrm{h}}\right)$, respectively. We denote the starting position of the road at the previous time stamp by $\left(x_{k-1}^{g, r}, y_{k-1}^{g, r}\right)$ and the current time stamp by $\left(x_{k}^{g, r}, y_{k}^{g, r}\right)$. These coordinates are illustrated in Figure 2. The traveled distance along the road is approximated as

$$
d_{k}=\sqrt{\left(x_{k}^{g, r}-x_{k-1}^{g, r}\right)^{2}+\left(y_{k}^{g, r}-y_{k-1}^{g, r}\right)^{2}} .
$$

Note that, it is assumed that the road starts at the rear-axle of the host vehicle. Due to short prediction lengths, typically around $0.5 \mathrm{~m}$, the starting point of the road at the current time can accurately be estimated using a linear approximation of the road. This approximation is used to find the point where the road intersects the $y$-axis of the local coordinate system at time $k$. As a result, the starting point of the road at time $k$ can be expressed as

$$
\left[\begin{array}{l}
x_{k}^{g, r} \\
y_{k}^{g, r}
\end{array}\right]=A^{-1} b
$$

where

$$
\begin{aligned}
A & =\left[\begin{array}{cc}
-\tan \left(\psi_{k-1}^{h}+\varphi_{k-1}\right) & 1 \\
-\tan \left(\psi_{k}^{h}+\frac{\pi}{2}\right) & 1
\end{array}\right] \\
b & =\left[\begin{array}{c}
y_{k-1}^{g, r}-x_{k-1}^{g, r} \tan \left(\psi_{k-1}^{h}+\varphi_{k-1}\right) \\
y_{k}^{h}-x_{k}^{h} \tan \left(\psi_{k}^{h}+\frac{\pi}{2}\right)
\end{array}\right] .
\end{aligned}
$$


Finally, we describe the process model of $y_{k}^{\text {off }}, \varphi_{k}$ and $\kappa_{0, k}$ as

$$
\begin{aligned}
y_{k+1}^{\mathrm{off}} & =\left[\begin{array}{ll}
-\sin \left(\psi_{k}^{\mathrm{h}}\right) & \cos \left(\psi_{k}^{\mathrm{h}}\right)
\end{array}\right]\left[\begin{array}{c}
x_{k}^{g, r}-x_{k}^{\mathrm{h}} \\
y_{k}^{g, r}-y_{k}^{\mathrm{h}}
\end{array}\right]+\nu_{k}^{y} \\
\varphi_{k+1} & =\varphi_{r}\left(d_{k}\right)-\left(\psi_{k}^{\mathrm{h}}-\psi_{k-1}^{\mathrm{h}}\right)+\nu_{k}^{\varphi} \\
\kappa_{0, k+1} & =\kappa_{r}\left(d_{k}\right)+\nu_{k}^{\kappa}
\end{aligned}
$$

where $\varphi_{r}(\cdot)$ and $\kappa_{r}(\cdot)$ are described by (14) and (15), respectively. Further, $\nu_{k}^{\kappa} \sim \mathcal{N}\left(0, \sigma_{\kappa}^{2}\right), \nu_{k}^{y} \sim \mathcal{N}\left(0, \sigma_{y}^{2}\right)$ and $\nu_{k}^{\varphi} \sim \mathcal{N}\left(0, \sigma_{\varphi}^{2}\right)$ are independent noise processes accounting for the uncertainties in the approximated quantities.

\section{B. Road fixed process model}

As previously stated, the road model consists of $n$ connected segments each describing the geometry of a specific fixed part of the road in front of the host vehicle.

When the host vehicle drives over a segment joint, the passed segment is removed and a new segment is appended at the end. As such, the curvature change rates are shifted to the left to make room for the new segment, i.e., $i_{j}:=i_{j+1} \forall j=$ $1 \ldots N-1$ and $i_{N}:=i_{N}+1$. The curvature change rate for the new segment is modelled as

$$
\kappa_{1}^{i_{N}}=\rho \frac{\kappa_{r}^{i_{N-1}}(L)}{L}+\nu_{k}^{\kappa_{1}}
$$

where $\nu_{k}^{\kappa_{1}} \sim \mathcal{N}\left(0, \sigma_{\kappa_{1}}^{2}\right)$ is a random variable describing the dynamics of the curvature change rate in the new segment The $\rho$-factor is used to model how much the road straightens after the appended segment. Setting this factor in the interval $[-1,0)$ will limit the curvature of the last segment and prevent the estimate to overshoot. For example, setting $\rho=-1$ would would result in an expected curvature at the end of the las segment that is zero (straight), i.e., $\mathbb{E}\left\{\kappa_{r}^{i_{N}}(L)\right\}=0$.

The time evolution of the width of the host vehicle lane and the distances to the left and right barrier are assumed to be constant with some small noise perturbation

$$
\begin{aligned}
& w_{k+1}^{\text {lane }}=w_{k}^{\text {lane }}+\nu_{k}^{w} \\
& d_{k+1}^{\mathrm{l}}=d_{k}^{\mathrm{l}}+\nu_{k}^{l} . \\
& d_{k+1}^{\mathrm{r}}=d_{k}^{\mathrm{r}}+\nu_{k}^{r} .
\end{aligned}
$$

where $\nu_{k}^{w} \sim \mathcal{N}\left(0, \sigma_{w}^{2}\right), \nu_{k}^{l} \sim \mathcal{N}\left(0, \sigma_{b}^{2}\right)$, and $\nu_{k}^{r} \sim \mathcal{N}\left(0, \sigma_{b}^{2}\right)$. Furthermore, as barriers can appear and disappear we need to describe the probability of these events. The former is modeled using a birth probability, $p_{b}$, and the latter with a probability of survival, $p_{s}$.

\section{Lane change compensation}

When the host vehicle changes lane, the road model needs to be translated to describe the middle of the new lane instead of the old one. The translation is performed by finding a parallel clothoid laterally adjusted by $\pm w_{k}^{\text {lane }}$, where the sign depends on the type of lane change. Using similar procedure as in [33], we can find a mapping that gives us a parallel clothoid with a lateral offset $\Delta y^{2}$. We denote this mapping as

$$
\left[\tilde{\mathbf{r}}_{k}, \tilde{\mathbf{b}}_{k} \tilde{\ell}_{k}^{i_{1}}, \ldots, \tilde{\ell}_{k}^{i_{N}}\right]=g\left(\mathbf{r}_{k}, \ell_{k}^{i_{1}}, \ldots, \ell_{k}^{i_{N}}, \Delta y\right)
$$

${ }^{2}$ In this case $\Delta y= \pm w_{k}^{\text {lane }}$ (depending in the lane change) but we define it more general here as we will need it later. where $\tilde{\mathbf{r}}_{k}$ is the road state of the translated road and $\tilde{\ell}_{k}^{i_{1}}, \ldots, \tilde{\ell}_{k}^{i_{N}}$ are the new segment lengths such that the roads are parallel and the segment transitions are still at the same place on the road. More detail on this mapping is given in Appendix B.

\section{Measurement Models}

In this section we present the measurements models used for the different information sources, i.e., lane marking measurements, estimates of moving vehicles and stationary road-side observations.

\section{A. Lane marking observations}

As discussed in [32], the polynomial parameter space is not suitable for fusion. However, a sufficient, and more convenient description of the information in the lane marking measurements is obtained by sampling the polynomials (1) and (2) at four points. Thus, we propose to sample the left and right lane marking polynomials at four points chosen equidistantly from 0 to $x_{\max }^{1}$ or $x_{\max }^{\mathrm{r}}{ }^{3}$, respectively. Let us denote a concatenated vector of the samples from left lane as $\mathbf{p}_{k}^{1}$ and from right lane as $\mathbf{p}_{k}^{\mathrm{r}}$ and the corresponding sampling distances as $\mathbf{s}_{k}^{1}$ and $\mathbf{s}_{k}^{\mathbf{r}}$.

For the road state to describe the lane geometry of the left and right lane markings as given by the camera, it needs to be translated by half a lane width to the left or to the right, respectively. Denoting $\mathbf{r}_{k}$ translated by $-w_{k}^{\text {lane }} / 2$ using (26) as $\tilde{\mathbf{r}}_{k}^{1}$ and similarly $\mathbf{r}_{k}$ translated by $w_{k}^{\text {lane }} / 2$ as $\tilde{\mathbf{r}}_{k}^{\mathrm{r}}$, the sampled lane marking polynomial can be described as

$$
\begin{aligned}
& \mathbf{p}_{k}^{1}=\tilde{\mathbf{c}}_{\tilde{r}}^{1}\left(\mathbf{s}_{k}^{1}\right)+\boldsymbol{\eta}_{k}^{1}\left(\mathbf{s}_{k}^{1}\right) \\
& \mathbf{p}_{k}^{\mathrm{r}}=\tilde{\mathbf{c}}_{\tilde{r}}^{\mathrm{r}}\left(\mathbf{s}_{k}^{\mathrm{r}}\right)+\boldsymbol{\eta}_{k}^{\mathrm{r}}\left(\mathbf{s}_{k}^{\mathrm{r}}\right)
\end{aligned}
$$

where $\tilde{\mathbf{c}}_{\tilde{r}}^{1}(\cdot)$ and $\tilde{\mathbf{c}}_{\tilde{r}}^{\mathrm{r}}(\cdot)$ is the position of the left and right lane markings at the given distances along the road and $\boldsymbol{\eta}_{k}^{1}\left(\mathbf{s}_{k}^{1}\right) \sim \mathcal{N}\left(\mathbf{0}, \mathbf{R}_{k}^{1}\left(\mathbf{s}_{k}^{1}\right)\right)$ and $\boldsymbol{\eta}_{k}^{\mathrm{r}}\left(\mathbf{s}_{k}^{\mathrm{r}}\right) \sim \mathcal{N}\left(\mathbf{0}, \mathbf{R}_{k}^{\mathrm{r}}\left(\mathbf{s}_{k}^{\mathrm{r}}\right)\right)$ are distance dependent measurement noise processes.

\section{B. Moving vehicle observations}

A vehicle traveling on a road has two main options; either it follows its lane or it changes lane by making a lane change or taking an exit. Vehicles following the road are in large respect having the same heading as the road at their current position. In this paper we want to use this fact, together with radar-camera observations on the heading of leading vehicles, to gain knowledge about the road geometry.

Given that the $j$ th observed vehicle is following its lane, its expected heading observation can be described as

$$
\phi_{k}^{j}=\varphi_{r}\left(s\left(x_{k}^{j}, y_{k}^{j}\right)\right)+\eta_{k}^{\phi}\left(s\left(x_{k}^{j}, y_{k}^{j}\right)\right)
$$

where $\varphi_{r}(\cdot)$ is given by (14), $s\left(x_{k}^{j}, y_{k}^{j}\right)$ is the arc length to the point on the road that is closest to the vehicle and $\eta_{k}^{\phi}\left(s\left(x_{k}^{j}, y_{k}^{j}\right)\right) \sim \mathcal{N}\left(0, \sigma_{\phi}^{2}\left(s\left(x_{k}^{j}, y_{k}^{j}\right)\right)\right)$ is a distance dependent measurement noise process.

Note that (29) is a linear function of the state $\mathbf{r}_{k}$ and is only valid for vehicles following their lane. How we treat vehicles not following their lane is covered in Section VI.

\footnotetext{
${ }^{3}$ Recall that $x_{\max }^{\mathrm{l}}$ or $x_{\max }^{\mathrm{r}}$ are given by the sensor as the maximum distance that the polynomial accurately describe the left and right lane geometry.
} 


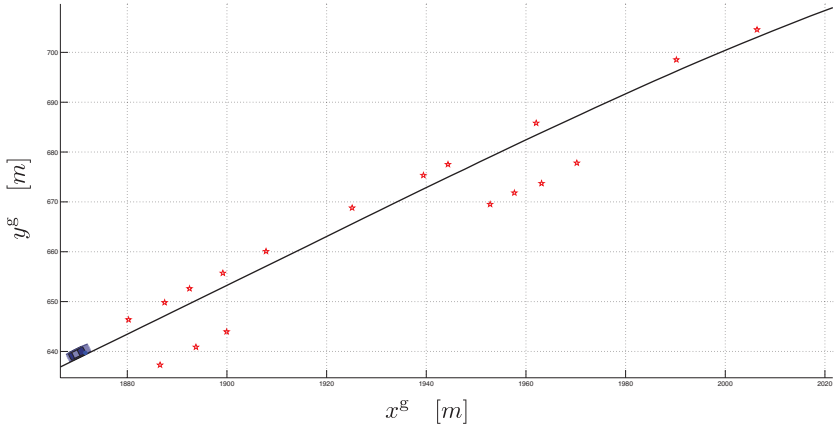

Figure 4. Radar observations of guard-rail posts (red stars) together with the true centerline of the host vehicle's lane (black) and host vehicle position (blue car).

\section{Stationary observations}

The radar sensor delivers observations on the position of stationary objects that reflect radar energy. Examples of such objects are vegetation, banks on the side of the road or the posts holding the guard-rails. In fact, when present, the guardrail posts are quite strong radar reflectors which also give information regarding the geometry of the road as shown in Figure 4. Obviously, there is information in these guard-rail measurements that we can exploit in order to better estimate thew shape of the road.

The stationary measurement vector, $\mathbf{z}_{k}^{\mathrm{s}}$, can thus contain informative observations from the left and right guard-rail, we denote these $\mathbf{z}_{k}^{1}$ and $\mathbf{z}_{k}^{\mathrm{r}}$, respectively, as well as less informative non-barrier measurements, we denote these as $\mathbf{z}_{k}^{\mathrm{nb}}$.

Unfortunately, the radar observations that we receive are not labeled as barrier or non-barrier measurements. To handle this data association uncertainty we propose to introduce the data association hypothesis set $\mathcal{H}$. Each hypothesis $\mathcal{H}^{i} \in \mathcal{H}$ is defined as the 4-tuple

$$
\mathcal{H}^{i}=\left\langle\boldsymbol{\lambda}^{i}, n_{1}^{i}, n_{\mathrm{r}}^{i}, n_{\mathrm{nb}}^{i}\right\rangle
$$

where $\boldsymbol{\lambda}^{i}$ is a $m_{k} \times 1$ data association vector, where $m_{k}$ is the number of observations, and $n_{1}^{i}, n_{\mathrm{r}}^{i}$ and $n_{\mathrm{nb}}^{i}$ are the number of left, right and non-barrier observations, respectively. Given the data association vector, the vector, $\mathbf{z}_{k}^{\mathrm{s}}$ can be sorted into three sub-vectors containing observations on the right barrier, $\mathbf{z}_{k}^{\mathrm{r}}$, the left barrier, $\mathbf{z}_{k}^{1}$ and non-barrier, $\mathbf{z}_{k}^{\mathrm{nb}}$. In the following sections we derive the conditional models for these vectors.

1) Guard-rail observations: Note that in [17], we only considered the lateral displacement and resorted to rules and heuristics to account for the longitudinal distribution. In this paper we instead propose a complete statistical model covering both dimensions. For the sake of brevity, we present the model for a single generic (left or right) barrier measurement vector denoted $\mathbf{z}_{k}^{\mathrm{b}}$, as the descriptions of left and right barrier are completely analogous. Further, without loss of generality, we assume that the measurements in $\mathbf{z}_{k}^{\mathrm{b}}$ are sorted in ascending longitudinal position along the barrier.

The probabilistic relationship between guard-rail observations and the road is more easily modelled with respect to the longitudinal distance and lateral displacement along the road.
Let us introduce $v_{k}^{i}$ and $s_{k}^{i}$ as the lateral and longitudinal coordinate of barrier measurement $\mathbf{z}_{k}^{\mathrm{b}, i}$ in a curved coordinate system aligned with the barrier. In this curved coordinate frame, the lateral position of the $i$ th barrier observation can simply be modeled as

$$
v_{k}^{i}=\eta_{k}^{\mathrm{v}, i}
$$

where $\eta_{k}^{\mathrm{v}, i} \sim \mathcal{N}\left(0, \sigma_{\mathrm{v}}^{2}\right)$ model lateral measurement uncertainty. That is, we model the barrier measurements as being on the barrier and lateral deviations are modeled as measurement noise.

The longitudinal distribution of the barrier observations is, however, a bit more intricate. Clearly it dependents on the probability of detecting each individual guard-rail post and, consequently, how much of the barrier that is visible to the radar. Let $\left[s_{\min }, s_{\max }\right]$ be the interval ${ }^{4}$ for which the barrier is in the field-of-view of the sensor. Further, let us assume that the guard-rail posts are positioned every $\delta p$ meters and that each post is detected independently with probability $P_{D}$. Using this, the longitudinal coordinate of the $i$ th barrier measurement is described as

$$
s_{k}^{i}=s_{k}^{i-1}+m_{i} \delta p+\eta_{k}^{s, i}
$$

where $s_{k}^{0}=s_{\min }, m_{i} \sim \operatorname{Geo}\left(P_{D}\right)$ is a geometrically distributed random variable modeling the number of guard-rail posts that pass between consecutive detection by the radar and $\eta_{k}^{\mathrm{s}, i} \sim \mathcal{N}\left(0, \sigma_{\mathrm{s}}^{2}\right)$ models measurement noise uncertainty as well as uncertainty in $\delta p$. As a consequence of the geometrical distribution of $m_{i}$, the number of barrier measurements, $n_{\mathrm{b}}$, is binomial distributed according to

$$
n_{\mathrm{b}} \sim \operatorname{Binomial}\left(\left\lfloor l_{k}^{\mathrm{b}} / \delta p\right\rfloor, P_{D}\right)
$$

where $|\cdot|$ is the floor function and $l_{k}^{\mathrm{b}}$ is the visible length of the barrier. From this procedure we can describe the longitudinal position of $n_{\mathrm{b}}$ barrier observations in the curved road-aligned coordinate system.

Being able to describe the $i$ th barrier measurement in the barrier aligned coordinates, $v_{k}^{i}$ and $s_{k}^{i}$, we now want to describe in the local coordinate system, $\mathbf{z}_{k}^{\mathrm{b}, i}$. The longitudinal axis of the barrier aligned coordinate system is given in the local coordinate system by (13) parameterized by a barrier road state, denoted $\mathbf{r}_{k}^{\mathrm{b}}$. This barrier road state is found by translating $\mathbf{r}_{k}$ using (26) with $\Delta y$ set to either $d_{k}^{\mathrm{l}}$ or $d_{k}^{\mathrm{r}}$ depending on which barrier to describe. Further, let us denote the resulting barrier clothoid as $\tilde{\mathbf{c}}_{\mathbf{r}}^{\mathrm{b}}(s)$, i.e. the clothoid parameterized with $\mathbf{r}_{k}^{\mathrm{b}}$. Using the resulting barrier aligned coordinate system, $\mathbf{z}_{k}^{\mathrm{b}, i}$ can now be described as

$$
\mathbf{z}_{k}^{\mathrm{b}, i}=\tilde{\mathbf{c}}_{r}^{\mathrm{b}}\left(s_{k}^{i}\right)+\operatorname{rot}\left(\varphi_{r}\left(s_{k}^{i}\right)\right)\left[\begin{array}{c}
\varepsilon_{k}^{i, \mathrm{~s}} \\
\varepsilon_{k}^{i, \mathrm{v}}+v_{k}^{i}
\end{array}\right]
$$

where $\operatorname{rot}(\cdot)$ is a 2D-rotation matrix and $\varphi_{r}(s)$ is the heading of the road at distance $s$ given by (14). The terms $\varepsilon_{k}^{i, \mathrm{~s}} \sim \mathcal{N}\left(0, \sigma_{\varepsilon}^{2}\right)$ and $\varepsilon_{k}^{i, \mathrm{v}} \sim \mathcal{N}\left(0, \sigma_{\varepsilon}^{2}\right)$ are introduced to

\footnotetext{
${ }^{4}$ These limits can be found by transforming respective barrier description to polar coordinates (sensor coordinates) and threshold w.r.t. the field-of-view of the sensor.
} 
describe modeling and approximation errors in the transform and is much smaller then both $\eta_{k}^{\mathrm{v}, i}$ and $\eta_{k}^{\mathrm{s}, i}$.

From (34) we can relate the measurement to its barrier aligned coordinates. To describe the measurement model, however, we need to describe the likelihood of the road and barrier state given the barrier measurements, i.e., $p\left(\mathbf{z}_{k}^{\mathrm{b}} \mid \mathbf{r}_{k}, \mathbf{b}_{k}, \mathcal{H}^{j}\right)$. If we, for brevity, omit conditioning on the data association hypothesis except the number of barrier measurements $n_{\mathrm{b}}^{j}$, this likelihood can be expressed as,

$$
\begin{aligned}
p & \left(\mathbf{z}_{k}^{\mathrm{b}} \mid n_{\mathrm{b}}^{j}, \mathbf{r}_{k}, \mathbf{b}_{k}\right) \\
= & \int p\left(\mathbf{z}_{k}^{\mathrm{b}} \mid \mathbf{s}_{k}, \mathbf{v}_{k}, \mathbf{r}_{k}, \mathbf{b}_{k}\right) p\left(\mathbf{s}_{k}, \mathbf{v}_{k} \mid n_{\mathrm{b}}^{j}, \mathbf{r}_{k}, \mathbf{b}_{k}\right) d \mathbf{s}_{k} d \mathbf{v}_{k} \\
= & \int p\left(\mathbf{z}_{k}^{\mathrm{b}} \mid \mathbf{s}_{k}, \mathbf{v}_{k}, \mathbf{r}_{k}, \mathbf{b}_{k}\right) p\left(\mathbf{v}_{k}\right) p\left(\mathbf{s}_{k} \mid n_{\mathrm{b}}^{j}, \mathbf{r}_{k}, \mathbf{b}_{k}\right) d \mathbf{s}_{k} d \mathbf{v}_{k} \\
= & \int\left[\prod_{i} \int p\left(\mathbf{z}_{k}^{\mathrm{b}, i} \mid s_{k}^{i}, v_{k}^{i}, \mathbf{r}_{k}, \mathbf{b}_{k}\right) p\left(v_{k}^{i}\right) d v_{k}^{i}\right] \\
& \times p\left(\mathbf{s}_{k} \mid n_{\mathrm{b}}^{j}, \mathbf{r}_{k}, \mathbf{b}_{k}\right) d \mathbf{s}_{k}
\end{aligned}
$$

where $\mathbf{s}_{k}=\left[s_{k}^{1}, \ldots, s_{k}^{n_{\mathrm{b}}^{j}}\right]^{T}$ and $\mathbf{v}_{k}=\left[v_{k}^{1}, \ldots, v_{k}^{n_{\mathrm{b}}^{j}}\right]^{T}$. Additionally, $p\left(\mathbf{z}_{k}^{\mathrm{b}, i} \mid s_{k}^{i}, v_{k}^{i}, \mathbf{r}_{k}, \mathbf{b}_{k}\right)$ is given by (34) and $p\left(v_{k}^{i}\right)$ by (31). The latter part, $p\left(\mathbf{s}_{k} \mid n_{\mathrm{b}}^{j}, \mathbf{r}_{k}, \mathbf{b}_{k}\right)$, can be expressed using Bayes' rule,

$$
p\left(\mathbf{s}_{k} \mid n_{\mathrm{b}}^{j}, \mathbf{r}_{k}, \mathbf{b}_{k}\right)=\frac{\operatorname{Pr}\left\{n_{\mathrm{b}}^{j} \mid \mathbf{s}_{k}\right\} p\left(\mathbf{s}_{k} \mid \mathbf{r}_{k}, \mathbf{b}_{k}\right)}{\operatorname{Pr}\left\{n_{\mathrm{b}}^{j} \mid \mathbf{r}_{k}, \mathbf{b}_{k}\right\}} .
$$

where $p\left(\mathbf{s}_{k} \mid \mathbf{r}_{k}, \mathbf{b}_{k}\right)$ is given by (32) and $\operatorname{Pr}\left\{n_{\mathrm{b}}^{j} \mid \mathbf{r}_{k}, \mathbf{b}_{k}\right\}$ by (33). The remaining component, $\operatorname{Pr}\left\{n_{\mathrm{b}}^{j} \mid \mathbf{s}_{k}\right\}$, is simply one for all valid hypotheses, i.e., when the number of observations in $\mathbf{s}_{k}$ are $n_{\mathrm{b}}^{j}$, and zero otherwise.

To simplify the likelihood expression in (35) even further, we note that using (32)

$$
\begin{aligned}
\int p\left(\mathbf{z}_{k}^{\mathrm{b}, i} \mid\right. & \left.s_{k}^{i}, v_{k}^{i}, \mathbf{r}_{k}, \mathbf{b}_{k}\right) p\left(v_{k}^{i}\right) d v_{k}^{i} \\
= & \int \mathcal{N}\left(\mathbf{z}_{k}^{\mathrm{b}, i} ; \tilde{\mathbf{c}}_{r}^{\mathrm{b}}\left(s_{k}^{i}\right), \operatorname{rot}\left(\varphi_{r}\left(s_{k}^{i}\right)\right) \boldsymbol{\Sigma}_{\varepsilon} \operatorname{rot}\left(\varphi_{r}\left(s_{k}^{i}\right)\right)^{T}\right) \\
& \quad \times \mathcal{N}\left(v_{k}^{i} ; 0, \sigma_{v}^{2}\right) d v_{k}^{i} \\
= & \mathcal{N}\left(\mathbf{z}_{k}^{\mathrm{b}, i} ; \tilde{\mathbf{c}}_{r}^{\mathrm{b}}\left(s_{k}^{i}\right), \boldsymbol{\Sigma}_{\mathbf{z}}\left(s_{k}^{i}\right)\right)
\end{aligned}
$$

where $\boldsymbol{\Sigma}_{\varepsilon}=\operatorname{diag}\left\{\sigma_{\varepsilon}^{2}, \sigma_{\varepsilon}^{2}\right\}$ and

$$
\boldsymbol{\Sigma}_{\mathbf{z}}=\operatorname{rot}\left(\varphi_{r}\left(s_{k}^{i}\right)\right)\left[\begin{array}{ll}
\sigma_{\varepsilon}^{2} & 0 \\
0 & \sigma_{\varepsilon}^{2}+\sigma_{v}^{2}
\end{array}\right] \operatorname{rot}\left(\varphi_{r}\left(s_{k}^{i}\right)\right)^{T}
$$

Furthermore, as $\sigma_{\varepsilon}$ is very small, (37) is only significant whenever $\mathbf{z}_{k}^{\mathrm{b}, i}$ is very close to $f_{r, b}\left(s_{k}^{i}, 0\right)$, i.e., when $s_{k}^{i}$ is close to distance to the measurement along the barrier. Thus, we propose to introduce $\hat{s}_{k}^{i}$, as the closest longitudinal distance to the measurement, which is calculated as

$$
\hat{s}_{k}^{i}=\underset{s}{\operatorname{argmin}}\left\|\mathbf{z}_{k}^{\mathrm{b}, i}-\tilde{\mathbf{c}}_{\mathbf{r}}^{\mathrm{b}}(s)\right\| .
$$

Assuming that $\sigma_{\varepsilon}^{2}$ is sufficiently small we can approximate (37) as a Dirac-delta in the $s$-dimension and, consequently, the remaining integral in (35) can be approximated as

$$
\begin{aligned}
p\left(\mathbf{z}_{k}^{\mathrm{b}} \mid \mathbf{r}_{k}, \mathbf{b}_{k}\right) & \propto \\
& {\left[\prod_{i} \mathcal{N}\left(\mathbf{z}_{k}^{\mathrm{b}, i} ; \tilde{\mathbf{c}}_{r}^{\mathrm{b}}\left(s_{k}^{i}\right), \mathbf{\Sigma}_{\mathbf{z}}\left(\hat{s}_{k}^{i}\right)\right)\right] } \\
& \times p\left(\hat{\mathbf{s}}_{k} \mid n_{\mathrm{b}}^{j}, \mathbf{r}_{k}, \mathbf{b}_{k}\right)
\end{aligned}
$$

where $p\left(\hat{\mathbf{s}}_{k} \mid n_{\mathrm{b}}^{j}, \mathbf{r}_{k}, \mathbf{b}_{k}\right)$ is given by (37) using $\hat{\mathbf{s}}_{k}=\left[\hat{s}_{k}^{1}, \ldots, \hat{s}_{k}^{n_{\mathrm{b}}}\right]$. Further, the mean of each Gaussian in (39) is dependent on the related measurement according to (38). Consequently, each mean is always the point on the barrier which is closest to the respective measurement. As such, moving the measurement along the barrier will influence the Gaussians, i.e., the Gaussians are flat (uninformative) along the barrier (s-dimension) and does not depend on the variance in that dimension $\left(\sigma_{\varepsilon}^{2}\right)$. This is, however, a convenient form in order to use standard tools to perform the measurement update.

To conclude, the resulting barrier likelihood in (39) is divided into two parts, the first part capturing the lateral displacement of each measurement in relation to the barrier aligned coordinate system. Using (34), the corresponding measurement model can be formed as

$$
\mathbf{z}_{k}^{\mathrm{b}, i}=\tilde{\mathbf{c}}_{r}^{\mathrm{b}}\left(\hat{s}_{k}^{i}\right)+\operatorname{rot}\left(\varphi_{r}\left(\hat{s}_{k}^{i}\right)\right)\left[\begin{array}{c}
\varepsilon_{k}^{i, \mathrm{~s}} \\
\varepsilon_{k}^{i, \mathrm{v}}+v_{k}^{i}
\end{array}\right]
$$

The second part describes the longitudinal displacement along the barrier and is modelled by (32). Note that, (32) is mainly dependent on the state through how large part of the barrier is with in the field of view of the sensor. As a consequence, this factor can be viewed as more of a weighting factor promoting data association hypothesis where the barrier measurements are evenly distributed along the barrier.

2) Non-barrier observations: Assuming that the $j$ th stationary observation is generated by a non-barrier object, it can be described by

$$
\mathbf{z}_{k}^{s, j} \sim \operatorname{Unif}(F O V)
$$

where $F O V$ is the field-of-view of the radar having volume $V$. Further, the number of non-barrier observations are assumed to be Poisson distributed according to

$$
n_{\mathrm{nb}} \sim \operatorname{Poisson}\left(V \beta_{\mathrm{nb}}\right)
$$

where $\beta_{\mathrm{nb}}$ is the intensity of non-barrier observations.

\section{Posterior CALCUlation AND GAUSSiAn APPROXIMATION}

In this section we derive the sought posterior using the process model proposed in Section III and the observation models derived in Section V. However, in order to incorporate the information from the barrier observations we do not calculate $p\left(\mathbf{r}_{k+1} \mid \mathbf{z}_{1: k+1}\right)$ directly. Instead we calculate the joint posterior of the road state and the barrier state, $p\left(\mathbf{r}_{k+1}, \mathbf{b}_{k+1} \mid \mathbf{z}_{1: k+1}\right)$. To calculate this posterior, we assume 
that we have a joint posterior from the previous time instance approximated as a Gaussian hierarchical model on the form

$$
\begin{aligned}
& p\left(\mathbf{r}_{k}, \mathbf{b}_{k} \mid \mathbf{z}_{1: k}\right) \approx \\
& {\left[\delta\left[e_{k}^{1}\right] \delta\left[e_{k}^{\mathrm{r}}\right]\left(1-p_{k \mid k}^{1}\right)\left(1-p_{k \mid k}^{\mathrm{r}}\right) \mathcal{N}\left(\mathbf{r}_{k} ; \hat{\mathbf{r}}_{k \mid k}, \mathbf{P}_{k \mid k}\right)\right]} \\
& +\left[\delta\left[\neg e_{k}^{1}\right] \delta\left[e_{k}^{\mathrm{r}}\right] p_{k \mid k}^{1}\left(1-p_{k \mid k}^{\mathrm{r}}\right)\right. \\
& \left.\quad \times \mathcal{N}\left(\left[\mathbf{r}_{k}, d_{k}^{\mathrm{l}}\right]^{T} ;\left[\hat{\mathbf{r}}_{k \mid k}, \hat{d}_{k \mid k}^{1}\right]^{T}, \mathbf{P}_{k \mid k}^{1}\right)\right] \\
& +\left[\delta\left[e_{k}^{1}\right] \delta\left[\neg e_{k}^{\mathrm{r}}\right]\left(1-p_{k \mid k}^{1}\right) p_{k \mid k}^{\mathrm{r}}\right. \\
& \left.\quad \times \mathcal{N}\left(\left[\mathbf{r}_{k}, d_{k}^{\mathrm{r}}\right]^{T} ;\left[\hat{\mathbf{r}}_{k \mid k}, \hat{d}_{k \mid k}^{\mathrm{r}}\right]^{T}, \mathbf{P}_{k \mid k}^{\mathrm{r}}\right)\right] \\
& +\left[\delta\left[\neg e_{k}^{1}\right] \delta\left[\neg e_{k}^{\mathrm{r}}\right] p_{k \mid k}^{1} p_{k \mid k}^{\mathrm{r}}\right. \\
& \left.\quad \times \mathcal{N}\left(\left[\mathbf{r}_{k}, d_{k}^{\mathrm{l}}, d_{k}^{\mathrm{r}}\right]^{T} ;\left[\hat{\mathbf{r}}_{k \mid k}, \hat{d}_{k \mid k}^{1}, \hat{d}_{k \mid k}^{\mathrm{r}}\right]^{T}, \mathbf{P}_{k \mid k}^{\mathrm{r}}\right)\right]
\end{aligned}
$$

where $\hat{\mathbf{r}}_{k \mid k}, \mathbf{P}_{k \mid k}$ is the mean and covariance of the road state given all measurements up to time $k$, Similarly, $\hat{d}_{k \mid k}^{\mathrm{l}}$ and $\hat{d}_{k \mid k}^{\mathrm{r}}$ are the mean position of the left and right barrier, respectively, and $\mathbf{P}_{k \mid k}^{\mathrm{l}}, \mathbf{P}_{k \mid k}^{\mathrm{r}}$ and $\mathbf{P}_{k \mid k}^{\mathrm{lr}}$ are the covariances for the road state extended with distance to left, right and both barriers. Further, $\delta[\cdot]$ is the Kronecker delta and $p_{k \mid k}^{1}$ and $p_{k \mid k}^{\mathrm{r}}$ are the probabilities that there exist a barrier to the left and the the right, respectively, given all previous observations.

The calculation of the updated pdf, $p\left(\mathbf{r}_{k+1}, \mathbf{b}_{k+1} \mid \mathbf{z}_{1: k+1}\right)$, and updated existence probabilities is performed in two steps, first a prediction step as described in Section VI-A followed by an observation specific update step described in Sections VI-B - VI-D.

\section{A. Predicted density}

The predicted density, $p\left(\mathbf{r}_{k+1}, \mathbf{b}_{k+1} \mid \mathbf{z}_{1: k}\right)$ is calculated using the Chapman-Kolmogorov equation:

$$
\begin{aligned}
p\left(\mathbf{r}_{k+1}, \mathbf{b}_{k+1} \mid \mathbf{z}_{1: k}\right) & =\sum_{e_{k}^{1}, e_{k}^{\mathrm{r}}} \int p\left(\mathbf{r}_{k+1}, \mathbf{b}_{k+1} \mid \mathbf{r}_{k}, \mathbf{b}_{k}\right) \\
& \times p\left(\mathbf{r}_{k}, \mathbf{b}_{k} \mid \mathbf{z}_{1: k}\right) \mathrm{d} \mathbf{r}_{k} \mathrm{~d} d_{k}^{1} \mathrm{~d} d_{k}^{\mathrm{r}}
\end{aligned}
$$

where $p\left(\mathbf{r}_{k+1}, \mathbf{b}_{k+1} \mid \mathbf{r}_{k}, \mathbf{b}_{k}\right)$ is the transition density defined by the state process model described in Section IV. Note that, due to the nonlinearities in, e.g., $\tilde{\mathbf{c}}_{r}(\cdot)$, there is no analytical solution to (44). Instead we resort to a Gaussian approximation using the square-root Unscented Transform (UT) [34] using the cubature rule [35].

Note that the resulting Gaussian approximation is on the same form as (43) but with predicted means, covariances and barrier existence probabilities. The existence probabilities of the left barrier is predicted using the birth and survival probabilities as

$$
p_{k+1 \mid k}^{1}= \begin{cases}p_{b}, & \text { if } e_{k}^{1}=0 \\ p_{s} p_{k \mid k}^{1}, & \text { if } e_{k}^{1}=1\end{cases}
$$

That is, if the barrier did not exist in the previous time instance, it exists now with probability $p_{b}$, and if it existed in the previous time instance, it will still be present with a probability reduced by a factor $p_{s}$. The existence probability of the right barrier is found analogously and the predicted probability of non-existence is simply the complement of (45).

\section{B. Lane measurement posterior}

Assuming that we have a Gaussian approximation of the predicted density in (44), we want to calculate an updated density using the geometry information in the lane marking polynomials given by the camera sensor. Additionally, we want to compensate the road state if the host vehicle has performed a lane change since the last lane marking observation. A lane change is easily identified by detecting jumps in the zerothorder coefficient of the lane marking polynomials in the order of $w_{k}^{\text {lane }}$. When detected, the translated road state is given by the procedure described in Section IV-C.

The posterior that is calculated using lane marking observations is found as a Gaussian approximation of

$$
\begin{aligned}
& p\left(\mathbf{r}_{k+1}, \mathbf{b}_{k+1} \mid \mathbf{z}_{1: k}, \mathbf{z}_{k+1}^{1}, \mathbf{z}_{k+1}^{\mathrm{r}}\right) \propto \\
& p\left(\mathbf{z}_{k+1}^{1} \mid \mathbf{r}_{k+1}\right) p\left(\mathbf{z}_{k+1}^{\mathrm{r}} \mid \mathbf{r}_{k+1}\right) p\left(\mathbf{r}_{k+1}, \mathbf{b}_{k+1} \mid \mathbf{z}_{1: k}\right)
\end{aligned}
$$

where the likelihoods $p\left(\mathbf{z}_{k+1}^{1} \mid \mathbf{r}_{k+1}\right)$ and $p\left(\mathbf{z}_{k+1}^{\mathrm{r}} \mid \mathbf{r}_{k+1}\right)$ are given by (27) and (28), respectively. Note that since the likelihoods are non-linear we use the update step in the squareroot CKF [35] to perform the Gaussian approximation of (46).

\section{Moving vehicle posterior}

As mentioned in Section V-B we only consider the vehicles that are deemed to follow their lane. To determine which vehicles are following their lane we use gating, where the Mahalanobis distance between the measurement heading of the vehicle is compared to the predicted heading of the road at the same location. If this distance is greater than a threshold, in our case 2.25 , the vehicle is classified as not following the road and not considered in the update.

Observations passing through the gate are used to update the predicted density as

$$
\begin{aligned}
& p\left(\mathbf{r}_{k+1}, \mathbf{b}_{k+1} \mid \mathbf{z}_{1: k}, \mathbf{z}_{k+1}^{\mathrm{v}}\right) \propto \\
& \prod_{j \in \mathcal{G}} p\left(\phi_{k+1}^{j} \mid \mathbf{r}_{k+1}\right) p\left(\mathbf{r}_{k+1}, \mathbf{b}_{k+1} \mid \mathbf{z}_{1: k}\right)
\end{aligned}
$$

where $\mathcal{G}$ is the set of observation indices passing through the gate and $p\left(\phi_{k+1}^{j} \mid \mathbf{r}_{k+1}\right)$ is defined in (29), Note that (29) is a linear function of the state and, as such, can be solved exactly using the Kalman filter update equations.

\section{Stationary observations posterior}

To calculate the posterior using the stationary observations we need to consider the data association uncertainty regarding which observations originated from the left and right barrier and which are non-barrier measurements. To handle this uncertainty we introduced the hypothesis set $\mathcal{H}$ in Section $\mathrm{V}-\mathrm{C}$. The posterior can, thus, be calculated as

$$
\begin{aligned}
& p\left(\mathbf{r}_{k+1}, \mathbf{b}_{k+1} \mid \mathbf{z}_{1: k}, \mathbf{z}_{k+1}^{\mathrm{s}}\right)= \\
& \quad \sum_{\mathcal{H}^{i} \in \mathcal{H}} p\left(\mathbf{r}_{k+1}, \mathbf{b}_{k+1} \mid \mathcal{H}^{i}, \mathbf{z}_{1: k}, \mathbf{z}_{k+1}^{\mathrm{s}}\right) \operatorname{Pr}\left\{\mathcal{H}^{i} \mid \mathbf{z}_{1: k}, \mathbf{z}_{k+1}^{\mathrm{s}}\right\},
\end{aligned}
$$

where $p\left(\mathbf{r}_{k+1}, \mathbf{b}_{k+1} \mid \mathcal{H}^{i}, \mathbf{z}_{1: k+1}\right)$ is the conditional posterior having no data association uncertainty and $\operatorname{Pr}\left\{\mathcal{H}^{i} \mid \mathbf{z}_{1: k+1}\right\}$ is the posterior probability of the $i$ th hypothesis. Below we derive the expressions of each of these posteriors. 
1) Conditional posterior: Using the data association vector $\lambda^{i}$ we can partition the stationary observations, $\mathbf{z}_{k+1}^{\mathrm{s}}$, into the three sub-vectors, $\mathbf{z}_{k+1}^{1}, \mathbf{z}_{k+1}^{\mathrm{r}}$ and $\mathbf{z}_{k+1}^{\mathrm{nb}}$. Using these vectors and Bayes' rule the conditional posterior can be expressed as

$$
\begin{aligned}
& p\left(\mathbf{r}_{k+1}, \mathbf{b}_{k+1} \mid \mathcal{H}^{i}, \mathbf{z}_{1: k}, \mathbf{z}_{k+1}^{\mathrm{s}}\right) \propto p\left(\mathbf{z}_{k+1}^{1} \mid \mathbf{r}_{k+1}, \mathbf{b}_{k+1}, \mathcal{H}^{i}\right) \\
& \quad \times p\left(\mathbf{z}_{k+1}^{\mathrm{r}} \mid \mathbf{r}_{k+1}, \mathbf{b}_{k+1}, \mathcal{H}^{i}\right) p\left(\mathbf{z}_{k+1}^{\mathrm{nb}} \mid \mathcal{H}^{i}\right) p\left(\mathbf{r}_{k+1}, \mathbf{b}_{k+1} \mid \mathbf{z}_{1: k}\right) .
\end{aligned}
$$

That is, the conditional posterior is updated using three independent likelihoods, where $p\left(\mathbf{z}_{k+1}^{\mathrm{nb}} \mid \mathcal{H}^{i}\right)=\prod_{j} p\left(\mathbf{z}_{k+1}^{\mathrm{nb}, j} \mid \mathcal{H}^{i}\right)$ is given by (41) and is just a scaling factor as its independent of the states.

For the barrier likelihoods in (49), the observation model in (40) is non-linear so (49) is approximated using the updatestep in the square-root CKF to calculate its first two moments. Further, the longitudinal likelihoods described by (32) is only dependent on the states through the visibility lengths of the barriers and in the filter implementation these are approximated using the visibility length of the mean state. As this dependence is weak, this approximation has little effect on the calculated conditional posterior.

2) Posterior hypothesis probability: To complete (48) we need to derive an expression for the posterior hypothesis probability. Using Bayes' rule,

$$
\operatorname{Pr}\left\{\mathcal{H}^{i} \mid \mathbf{z}_{1: k+1}\right\} \propto p\left(\mathbf{z}_{k+1} \mid \mathcal{H}^{i}, \mathbf{z}_{1: k}\right) \operatorname{Pr}\left\{\mathcal{H}^{i} \mid \mathbf{z}_{1: k}\right\}
$$

where the hypothesis likelihood

$$
\begin{aligned}
& p\left(\mathbf{z}_{k+1} \mid \mathcal{H}^{i}, \mathbf{z}_{1: k}\right)= \\
& \quad \int p\left(\mathbf{z}_{k+1} \mid \mathcal{H}^{i}, \mathbf{r}_{k+1}, \mathbf{b}_{k+1}\right) p\left(\mathbf{r}_{k+1}, \mathbf{b}_{k+1} \mid \mathbf{z}_{1: k}\right) \mathrm{d} \mathbf{r}_{k+1} \mathrm{~d} \mathbf{b}_{k+1}
\end{aligned}
$$

is approximated as a Gaussian density as an intermediate step in the sq-CKF approximation of (49).

Remaining is the prior on $\mathcal{H}^{i}$, which can be found by dividing the hypothesis set into its parts giving the following expression,

$$
\begin{aligned}
\operatorname{Pr}\left\{\mathcal{H}^{i} \mid \mathbf{z}_{1: k}\right\} & =\operatorname{Pr}\left\{\boldsymbol{\lambda}^{i}, n_{1}^{i}, n_{\mathrm{r}}^{i}, n_{\mathrm{nb}}^{i} \mid \mathbf{z}_{1: k}\right\} \\
& =\operatorname{Pr}\left\{\boldsymbol{\lambda}^{i} \mid n_{1}^{i}, n_{\mathrm{r}}^{i}, n_{\mathrm{nb}}^{i}\right\} \\
& \times \operatorname{Pr}\left\{n_{1}^{i} \mid \mathbf{z}_{1: k}\right\} \operatorname{Pr}\left\{n_{\mathrm{r}}^{i} \mid \mathbf{z}_{1: k}\right\} \operatorname{Pr}\left\{n_{\mathrm{nb}}^{i}\right\} .
\end{aligned}
$$

The first term in (52) is simply a combinatoric related to how many ways one can choose $n_{1}^{i}+n_{\mathrm{r}}^{i}+n_{\mathrm{nb}}^{i}$ terms of different types, i.e.,

$\operatorname{Pr}\left\{\boldsymbol{\lambda}^{i} \mid n_{\mathrm{b}}^{i}, n_{\mathrm{r}}^{i}, n_{\mathrm{nb}}^{i}\right\}=\left(\begin{array}{c}n_{\mathrm{b}}^{i}+n_{\mathrm{r}}^{i}+n_{\mathrm{nb}}^{i} \\ n_{\mathrm{b}}^{i}\end{array}\right)^{-1}\left(\begin{array}{c}n_{\mathrm{r}}^{i}+n_{\mathrm{nb}}^{i} \\ n_{\mathrm{r}}^{i}\end{array}\right)^{-1}$

Again, as $\operatorname{Pr}\left\{n_{\mathrm{b}}^{i} \mid \mathbf{z}_{1: k}\right\}$ and $\operatorname{Pr}\left\{n_{\mathrm{r}}^{i} \mid \mathbf{z}_{1: k}\right\}$ are similar, for brevity we express them using a the more generic $n_{\mathrm{b}}^{i}$ covering both models. The term $\operatorname{Pr}\left\{n_{\mathrm{b}}^{i} \mid \mathbf{z}_{1: k}\right\}$ can be partitioned as

$$
\operatorname{Pr}\left\{n_{\mathrm{b}}^{i} \mid \mathbf{z}_{1: k}\right\}=\sum_{\substack{\mathrm{b}, i \\ e_{k+1}}} \operatorname{Pr}\left\{n_{\mathrm{b}}^{i} \mid \mathbf{z}_{1: k}, e_{k+1}^{\mathrm{b}, i}\right\} \operatorname{Pr}\left\{e_{k+1}^{\mathrm{b}, i} \mid \mathbf{z}_{1: k}\right\}
$$

where $\operatorname{Pr}\left\{n_{\mathrm{b}}^{i} \mid \mathbf{z}_{1: k}, e_{k+1}^{\mathrm{b}, i}\right\}$ is approximately given by (33) for $e_{k+1}^{\mathrm{b}, i}=1$. On the other hand, if the barrier does not exist it is

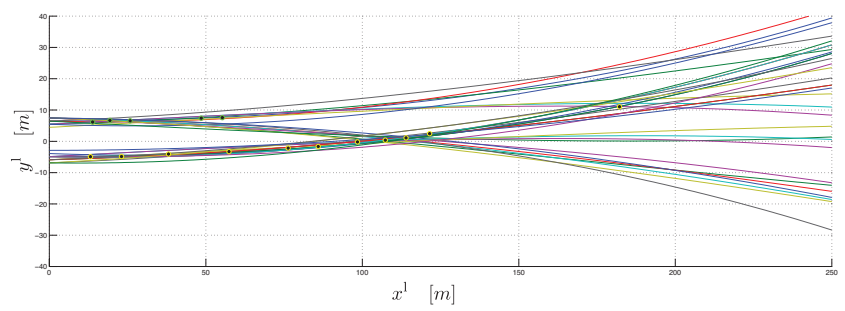

Figure 5. Results of the Hough-transform performed on the stationary observations (black stars). Each curve represents a cubic polynomial used to generate the hypothesis set $\mathcal{H}$.

one for $n_{\mathrm{b}}^{i}=0$ and zeros otherwise. The predicted existence probabilities in (54) is calculated as

$$
\operatorname{Pr}\left\{e_{k+1}^{\mathrm{b}, i} \mid \mathbf{z}_{1: k}\right\}= \begin{cases}p_{k+1 \mid k}^{\mathrm{b}} & , \text { if } e_{k+1}^{\mathrm{b}, i}=1 \\ \left(1-p_{k+1 \mid k}^{\mathrm{b}}\right) & , \text { if } e_{k+1}^{\mathrm{b}, i}=0\end{cases}
$$

Finally, the probability of number of clutter measurements $\operatorname{Pr}\left\{n_{\mathrm{nb}}^{i}\right\}$ is given by the model in (42).

3) Gaussian approximation: Using the procedure in Section VI-D1 and Section VI-D2 results in a Gaussian mixture approximation of (48). To return to the same form as the prior, we approximate the mixture with one Gaussian density having the same first two moments. Note that the posterior existence probability can be found by marginalizing out the other state variables from the posterior density in (48).

4) Hypothesis generation: Due to a large number of stationary obseravations, it is computationally intractable to consider the all hypotheses. To limit the hypotheses to only the most likely, we propose to segment the stationary observations using the Hough-transform [37].

To restrict the parameter span in the Hough-transform, we introduce three sub-segmentations, each targeting a specific type of hypothesis, namely, both barriers exist, only the left barrier exists and only the right barrier exists. To describe the shape of each candidate we use cubic polynomials in the local Cartesian coordinate frame. For single barrier hypotheses we use a single cubic polynomial and for dual barrier hypotheses we use two having separate zeroth order coefficients (offset) but sharing higher order coefficients.

The data association hypotheses are generated by selecting the Hough-candidates that received the highest vote in respective category. For each candidate, the observations intersecting the left or right Hough-curve are sorted as left or right barrier observations, respectively, and the remaining observations are selected as non-barrier. To ensure that all hypotheses are unique, candidates that generate an identical hypothesis to one already selected are removed. In total $n_{h}$ hypotheses are selected in each category. Figure 5 shows an example of the shape of the hypothesis generated using the Hough-transform.

\section{E. Filter structure and initialization}

The filter is initialized when the host vehicle speed is above $70 \mathrm{kph}$ and we receive observations on both left and right lane markings. The filter is initiated using a Gaussian prior $\hat{\mathbf{r}}_{0 \mid 0}$ and $\mathbf{P}_{0 \mid 0}$ and performing the update as described in Section VI-B. A summary of the algorithm is given in Algorithm 1. 


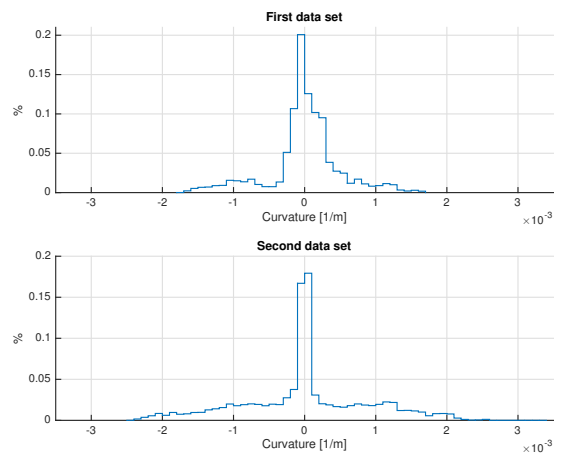

Figure 6. Normalized histogram of the curvatures in the first (above) and second (below) data set.

The algorithm is implemented in Matlab and is not optimized for speed. Executing the code on a MacBook Pro (early 2011) with a $2,7 \mathrm{GHz}$ Intel Core i7 resulted in the following computational times for the different parts: prediction $(4 \mathrm{~ms})$, update with lane marking measurements $(22 \mathrm{~ms})$, update with moving objects $(14 \mathrm{~ms})$ and update with stationary objects (103 ms). Clearly, the update with stationary objects is the most computationally demanding part of the algorithm mainly due to the non-linear measurement update and handling multiple hypothesis.

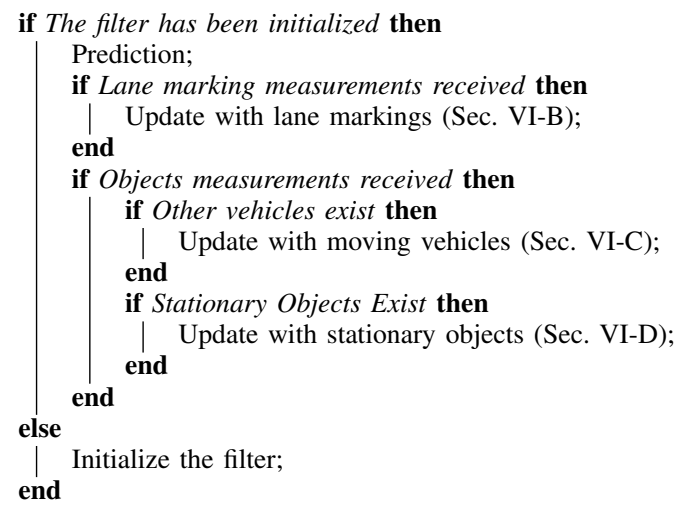

Algorithm 1: Pseudo-code of the filtering algorithm.

\section{EXPERIMENTAL RESULTS}

In this section we assess the performance of our filter on two sets of $\log$ files. The first includes measurements from highways where the road varies from straight to winding, which is the same set used for evaluations in [17]. The second set is recorded along a highway in a mountainous area. The data in this set is more curvy and presents some very challenging highway scenarios. A histogram over the curvatures in the different data sets can be viewed in Figure 6. We evaluate the estimation accuracy using combinations of our three observation sources, leading vehicles, stationary road side detections and lane marking measurements.

We use two measures of performance. Both measures are based on error in the estimated position of the road sampled every $20 \mathrm{~m}$ up to $200 \mathrm{~m}$ ahead of the host vehicle by calculating the Euclidian distance to the true position of the road at the same distances. The first measure is the root mean squared error (RMSE) where the squared error is calculated after each time step (update cycle) and at each distance and the RMSE is taken over time. As some ADAS applications are interested in positioning leading vehicles in or out of lane, in the second measure of performance, we calculate the percentage of time steps where the error is below an average lane width denoted $\omega$. For the calculations we assume that $\omega=3.5 \mathrm{~m}$. The same calculation has been performed for half a lane width. The ground truth of the road is obtained in [17].

\section{A. Parameter values}

The settings for the process and measurement noises are expressed in terms of their standard deviations.

- The process noise parameters are set to $\sigma_{y}=0.4[\mathrm{~m}]$, $\sigma_{\varphi}=0.5\left[^{\circ}\right], \sigma_{\kappa}=10^{-5}\left[\mathrm{~m}^{-1}\right], \sigma_{\kappa_{1}}=2 e-6\left[\mathrm{~m}^{-2}\right], \sigma_{\omega}=$ $0.0175[\mathrm{~m}], \sigma_{l}=1[\mathrm{~m}], \sigma_{r}=1[\mathrm{~m}], p_{b}=0.1$ and $p_{s}=$ 0.95 .

- For lane marking measurements with confidence higher than 2, the measurement noise for left and right lane marking samples are set analogously to $\mathbf{R}^{l}\left(\mathbf{s}_{k}^{l}\right)=$ $\operatorname{diag}\left(\left[\sigma_{x}, \sigma_{y}\left(s_{k}^{l, 1}\right), \sigma_{x}, \sigma_{y}\left(s_{k}^{l, 2}\right), \ldots\right]\right)$ where $\sigma_{x}=0.01[\mathrm{~m}]$ and $\sigma_{y}(s)=0.0175+s / 10[\mathrm{~m}]$. The measurement noise for the heading of a leading vehicle depends on the longitudinal distance of that vehicle to the host and is calculated by $\sigma_{\phi}(s)=1.75+1.5 \mathrm{~s} / 100\left[^{\circ}\right]$ and the gate size is set to $2.25 \sigma_{\phi}$. For the barriers we have $\sigma_{\varepsilon}=0.2[\mathrm{~m}], \sigma_{\mathrm{v}}=1[\mathrm{~m}], \beta_{n b} V=7.3$ and $n_{h}=8$.

- For the initialization, the parameters of $\hat{\mathbf{r}}_{0 \mid 0}$ are set to $y_{0}^{\text {off }}=0.5\left(l_{0}^{0}+r_{0}^{0}\right), \varphi_{0}=0.5\left(l_{0}^{1}+r_{0}^{1}\right), \kappa_{0,0}=0.5\left(l_{0}^{2}+r_{0}^{2}\right)$, $\kappa_{1}^{i_{1}}=0.5\left(l_{0}^{3}+r_{0}^{3}\right)$ and $w_{0}^{\text {lane }}=l_{0}^{0}-r_{0}^{0}$. Additionally, the parameters of the square root of $\mathbf{P}_{0 \mid 0}$ are set to $\sigma_{0}^{y}=$ $0.5[\mathrm{~m}], \sigma_{0}^{\varphi}=1.5\left[^{\circ}\right], \sigma_{0}^{\kappa}=0.0001\left[\mathrm{~m}^{-1}\right]$ and $\sigma_{0}^{\kappa_{1}}=0$.

\section{B. Evaluation results for the first data set}

In this section we use the first data set to evaluate the performance of our algorithm. The data contain both straight and curvy highways, where some of the drive tests are busy with traffic and others are not. Similarly, guard rails are present at only parts of the road.

Figure 7 depicts the comparison of the RMSE for the different distances taken over the whole data set, for three different scenarios: using only lane marking measurements, using measurements of lane markings and barriers and using measuremen ts of lane markings, moving vehicles and barriers. A comparison between the different cases reveals that the RMSE improves significantly when we combine barrier and lane marking measurements, additionally, the best performance is achieved when we use all three types of measurements.

For the case where we use all three types of measurements, the percentage of time that the error is below one lane width and below half a lane width is illustrated in Figure 8. We can see that the error at $200 \mathrm{~m}$ ahead of the host vehicle is below one lane width $89 \%$ of the times, and is below half a lane width $72 \%$ of the times. Moreover, we can see that at $100 \mathrm{~m}$ ahead, the error is below half a lane width $97 \%$ of the times.

Finally, Figure 9 depicts the comparison between the performance of our algorithm to the performance of the algorithm 


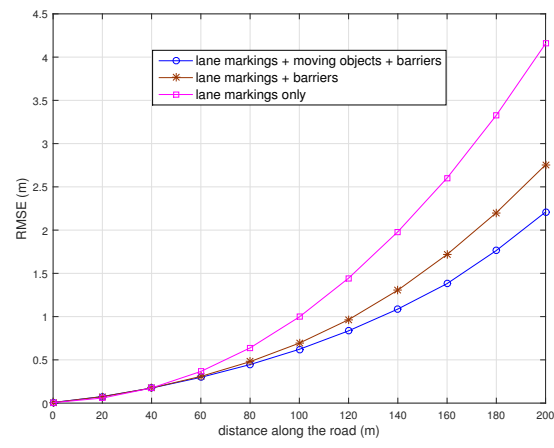

Figure 7. RMSE taken over the first data set.

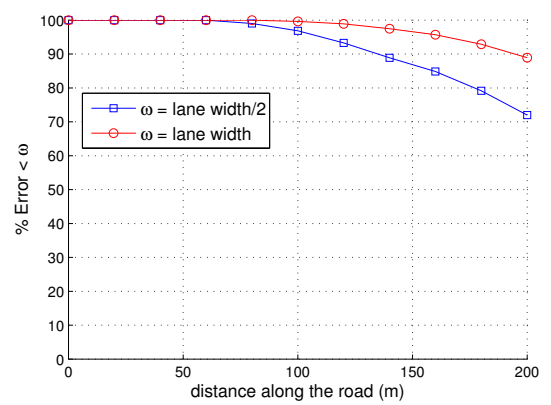

Figure 8. Percentage of time that the error is below a lane width and below half a lane width for the first data set. All three measurement types are used

presented in [17], for the case where all three measurement types have been used. As we can see, the developed algorithm outperforms the one presented in [17].

\section{Evaluation results for the second data set}

In this section we use the second data set to evaluate the performance of our filter. The measurements recorded in these tests are taken from extremely winding highways going through several tunnels. Particularly the tunnels proved to be a challenge, where the concrete tunnel walls act as mirrors for the radar signal giving rise to persistent detections that seem to be on the road surface and misleading our barrier model. Consequently, the RMSE significantly degrades due to this problem. Figure 10 illustrates the RMSE versus the distance

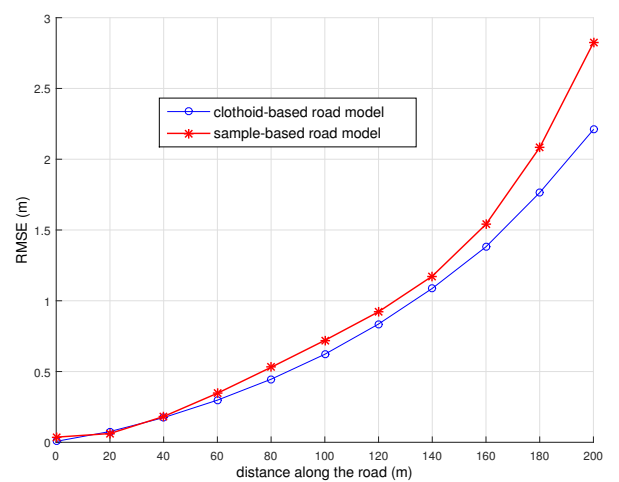

Figure 9. Comparison of RMSE (taken over the first data set) between our algorithm (clothoid-based) and the algorithm presented in [17] (sample-based).

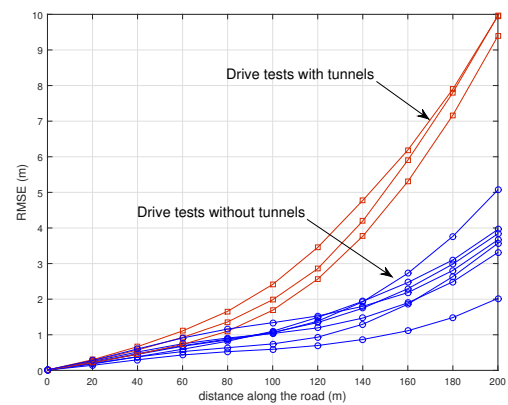

Figure 10. RMSE for the individual test drives in the second data set.

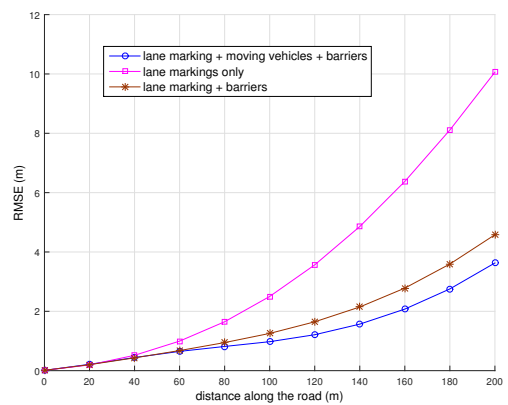

Figure 11. RMSE for the drive tests in the second data set which do not include tunnels.

along the road for different drive tests when the complete set of measurements are used to estimate the road. This figure clearly depicts the divide between the test drives that include tunnels and those that do not. This is a specific problem that could, for example, be tackled by using digital maps and GPS in order to locate the tunnels. Using such information we can shutdown the filter in tunnels and initialize it again after them. In addition, this map information can be used to improve the estimation of the road geometry if the GPS signal is accurate enough. To make the evaluation less dependent on the percentage of tunnels in the data set we have removed the $\log$ files containing the tunnels from the rest of the evaluation. Figure 11 illustrates the RMSE over all the remaining log files which do not include tunnels. Three cases are illustrated here, each depicting how including different types of measurements affects the error. Further, the percentage of time that the error is lower than a lane width and lower than half a lane width for the case where we use all three types of measurements, is depicted in Figure 12. We can see that $86 \%$ of the times the error at 200 meters is below a lane width and $63 \%$ of the times it is below half a lane width.

\section{CONCLUSIONS}

In this paper a filtering algorithm is derived to estimate the road geometry $200 \mathrm{~m}$ ahead of a host vehicle using the observations of lane markings, moving vehicles and barriers (guard rails, where the main focus is on highway scenarios. Two data sets, recorded in different types of highways, are used to evaluate the performance of the algorithm. The results indicate that road geometry estimation benefits from combining observations from camera and radar. Additionally, 


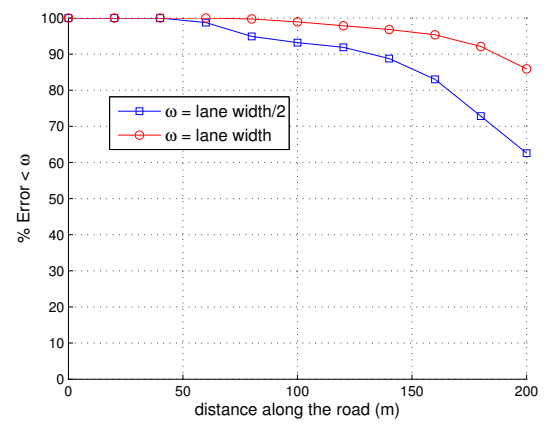

Figure 12. Percentage of the time that the error is below a lane width and below half a lane width for the drive tests of the second data set which do not include tunnels. All three measurement types are used.

barrier obseravtions, if present, significantly improve the road estimation performance for curvy highways.

The results also reveal that the filter is susceptible to structured noise present in the tunnels. There are different ways of alleviating this problem. First, one can use sensor observations to try to detect that we are in a tunnel and adjust the filter accordingly. Another alternative would be to make use of a satellite positioning sensor in combination with digital maps where the presence of tunnels are typically indicated. By knowing if we are in a tunnel we can trust the stationary radar observations less in these scenarios. Additionally, if we have access to digital maps we can use these directly as a prior in our road geometry filter.

\section{APPENDIX}

\section{A. Clothoid approximation}

In this paper we use a third-order polynomial Taylor approximation of the trigonometric functions in (7) around $s=L / 2$, where $L$ is the length of the segment. The resulting approximative expressions of the Cartesian coordinates of the road as a function of arc length $s$ is given

$$
\begin{aligned}
& \tilde{x}_{r}^{i}(s)=x_{0}^{i}+\left(\frac{S \dot{\alpha}^{3}}{24}-\frac{\kappa_{1} C \dot{\alpha}}{8}\right) s^{4} \\
& +\left(-\frac{L\left(\frac{S \dot{\alpha}^{3}}{6}-\frac{\kappa_{1} C \dot{\alpha}}{2}\right)}{2}-\frac{C \dot{\alpha}^{2}}{6}-\frac{\kappa_{1} S}{6}\right) s^{3} \\
& +\left(\frac{L \frac{C \dot{\alpha}^{2}+\kappa_{1} S}{2}}{2}-\frac{S \dot{\alpha}}{2}+\frac{3 L^{2}\left(\frac{S \dot{\alpha}^{3}}{6}-\frac{\kappa_{1} C \dot{\alpha}}{2}\right)}{8}\right) s^{2} \\
& +\left(C-\frac{L^{3}\left(\frac{S \dot{\alpha}^{3}}{6}-\frac{\kappa_{1} C \dot{\alpha}}{2}\right)}{8}-\frac{L^{2} \frac{C \dot{\alpha}^{2}+\kappa_{1} S}{2}}{4}+\frac{L S \dot{\alpha}}{2}\right) s \\
& \tilde{y}_{r}^{i}(s)=y_{0}^{i}+\left(-\frac{C \dot{\alpha}^{3}}{24}-\frac{\kappa_{1} S \dot{\alpha}}{8}\right) s^{4} \\
& +\left(\frac{L\left(\frac{C \dot{\alpha}^{3}}{6}+\frac{\kappa_{1} S \dot{\alpha}}{2}\right)}{2}-\frac{S \dot{\alpha}^{2}}{6}+\frac{\kappa_{1} C}{6}\right) s^{3}
\end{aligned}
$$

$$
\begin{aligned}
& +\left(\frac{C \dot{\alpha}}{2}+\frac{L \frac{S \dot{\alpha}^{2}-\kappa_{1} C}{2}}{2}-\frac{3 L^{2}\left(\frac{C \dot{\alpha}^{3}}{6}+\frac{\kappa_{1} S \dot{\alpha}}{2}\right)}{8}\right) s^{2} \\
& +\left(S+\frac{L^{3}\left(\frac{C \dot{\alpha}^{3}}{6}+\frac{\kappa_{1} S \dot{\alpha}}{2}\right)}{8}+\frac{L^{2} \frac{S \dot{\alpha}^{2}-\kappa_{1} C}{2}}{4}-\frac{L C \dot{\alpha}}{2}\right) s
\end{aligned}
$$

where

$$
\begin{aligned}
& S=\sin \left(\varphi_{0}^{i}+\kappa_{0}^{i} \frac{L}{2}+\kappa_{1}^{i} \frac{L^{2}}{8}\right) \\
& C=\cos \left(\varphi_{0}^{i}+\kappa_{0}^{i} \frac{L}{2}+\kappa_{1}^{i} \frac{L^{2}}{8}\right) \\
& \dot{\alpha}=\kappa_{0}^{i}+\kappa_{1}^{i} \frac{L}{2}
\end{aligned}
$$

Note that (56) and (57) are non-linear functions of the state dependent segment parameters, $\varphi_{0}^{i}, \kappa_{0}^{i}$, and $\kappa_{1}^{i}$.

\section{B. Clothoid translation}

The mapping (26) is based on the procedure in [33]. As such, the new road parameters of the parallel clothoid with lateral offset $\Delta y$ is approximated as

$$
\begin{aligned}
\tilde{\varphi}_{0} & =\varphi_{0} \\
\tilde{\kappa}_{0} & =\frac{1}{\frac{1}{\kappa_{0}}+\Delta y} \\
\tilde{\kappa}_{1}^{i} & =2 \frac{\Delta \varphi_{0}^{i}}{\left(\tilde{\ell}_{k}^{i}\right)^{2}}-2 \frac{\tilde{\kappa}_{0}^{i}}{\tilde{\ell}_{k}^{i}} \\
\tilde{\ell}_{k}^{i} & =\ell_{k}^{i}-\Delta y \Delta \varphi^{i}
\end{aligned}
$$

where $\Delta \varphi^{i}=\kappa_{0}^{i} \tilde{\ell}_{k}^{i}+\kappa_{1}^{i} / 2\left(\tilde{\ell}_{k}^{i}\right)^{2}$ and $\tilde{\kappa}_{0}^{i}=\tilde{\kappa}_{0}^{i-1}+\tilde{\kappa}_{1}^{i-1} \tilde{\ell}_{k}^{i-1}$.

The distance to the left and right barrier must also be adjusted to reflect the new position of the road by setting the new barrier distances to

$$
\begin{aligned}
& \tilde{d}_{k}^{\mathrm{l}}=d_{k}^{\mathrm{l}} \pm w_{k}^{\text {lane }} \\
& \tilde{d}_{k}^{\mathrm{r}}=d_{k}^{\mathrm{r}} \pm w_{k}^{\text {lane }}
\end{aligned}
$$

where positive shifts are for lane change to the right and negative are for lane change to the left.

\section{REFERENCES}

[1] J. C. McCall and M. M. Trivedi, "Video-based lane estimation and tracking for driver assistance: survey, system, and evaluation," Intelligent Transportation Systems, IEEE Transactions on, vol. 7, no. 1, pp. 20-37, 2006.

[2] B.-S. Shin, Z. Xu, and R. Klette, "Visual lane analysis and higher-order tasks: a concise review," Machine Vision and Applications, pp. 1-29, 2014.

[3] H. Loose, U. Franke, and C. Stiller, "Kalman particle filter for lane recognition on rural roads," in Intelligent Vehicles Symposium, 2009 IEEE, 2009, pp. 60-65.

[4] K. Kaliyaperumal, S. Lakshmanan, and K. Kluge, "An algorithm for detecting roads and obstacles in radar images," Vehicular Technology, IEEE Transactions on, vol. 50, no. 1, pp. 170-182, 2001.

[5] Y. Yamaguchi, M. Sengoku, and S. Motooka, "Using a van-mounted FM-CW radar to detect corner-reflector road-boundary markers," Instrumentation and Measurement, IEEE Transactions on, vol. 45, no. 4, pp. 793-799, 1996. 
6] C. Lundquist, L. Hammarstrand, and F. Gustafsson, "Road intensity based mapping using radar measurements with a probability hypothesis density filter," Signal Processing, IEEE Transactions on, vol. 59, no. 4, pp. 1397-1408, 2011.

[7] L. J. Quackenbush, I. Im, and Y. Zuo, "Road extraction: a review of lidar-focused studies," Remote Sensing of Natural Resources, pp. 155169, 2013.

[8] B. Yang, L. Fang, Q. Li, and J. Li, "Automated extraction of road markings from mobile lidar point clouds," Photogrammetric Engineering \& Remote Sensing, vol. 78, no. 4, pp. 331-338, 2012

[9] K. Peterson, J. Ziglar, and P. E. Rybski, "Fast feature detection and stochastic parameter estimation of road shape using multiple lidar," in Intelligent Robots and Systems, 2008. IROS 2008. IEEE/RSJ International Conference on. IEEE, 2008, pp. 612-619.

[10] W. S. Wijesoma, K. S. Kodagoda, and A. P. Balasuriya, "Road-boundary detection and tracking using ladar sensing," Robotics and Automation, IEEE Transactions on, vol. 20, no. 3, pp. 456-464, 2004.

[11] B. Ma, S. Lakshmanan, and A. O. Hero, "Simultaneous detection of lane and pavement boundaries using model-based multisensor fusion," Intelligent Transportation Systems, IEEE Transactions on, vol. 1, no. 3, pp. 135-147, 2000.

[12] Z. Zomotor and U. Franke, "Sensor fusion for improved vision based lane recognition and object tracking with range-finders," in Intelligent Transportation System, 1997. ITSC'97., IEEE Conference on. IEEE, 1997, pp. 595-600.

[13] A. Gern, U. Franke, and P. Levi, "Advanced lane recognition-fusing vision and radar," in Intelligent Vehicles Symposium, 2000. IV 2000. Proceedings of the IEEE. IEEE, 2000, pp. 45-51.

[14] A. Eidehall, J. Pohl, and F. Gustafsson, "Joint road geometry estimation and vehicle tracking," Control Engineering Practice, vol. 15, no. 12, pp. 1484-1494, 2007.

[15] U. Hofmann, A. Rieder, and E. D. Dickmanns, "Radar and vision data fusion for hybrid adaptive cruise control on highways," Machine Vision and Applications, vol. 14, no. 1, pp. 42-49, 2003

[16] C. Lundquist and T. B. Schön, "Joint ego-motion and road geometry estimation," Information Fusion, vol. 12, no. 4, pp. 253-263, 2011.

[17] Á. F. García-Fernández, L. Hammarstrand, M. Fatemi, and L. Svensson, "Bayesian road estimation using onboard sensors," Intelligent Transportation Systems, IEEE Transactions on, vol. 15, no. 4, pp. 1676-1689, Aug 2014.

[18] Y. Wang, L. Bai, and M. Fairhurst, "Robust road modeling and tracking using condensation," Intelligent Transportation Systems, IEEE Transactions on, vol. 9, no. 4, pp. 570-579, 2008

[19] Y. Wang, E. K. Teoh, and D. Shen, "Lane detection and tracking using b-snake," Image and Vision computing, vol. 22, no. 4, pp. 269-280, 2004.

[20] R. Aufrere, R. Chapuis, and F. Chausse, "A model-driven approach for real-time road recognition," Machine Vision and Applications, vol. 13, no. 2, pp. 95-107, 2001

[21] M. Bertozzi and A. Broggi, "Gold: A parallel real-time stereo vision system for generic obstacle and lane detection," Image Processing, IEEE Transactions on, vol. 7, no. 1, pp. 62-81, 1998.

[22] F. Bengtsson and L. Danielsson*, "A design architecture for sensor data fusion systems with application to automotive safety," in 15th world congress on ITS, 2008.

[23] L. Hammarstrand, M. Lundgren, and L. Svensson, "Adaptive radar sensor model for tracking structured extended objects," Aerospace and Electronic Systems, IEEE Transactions on, vol. 48, no. 3, pp. 1975-1995, 2012.

[24] L. Hammarstrand, L. Svensson, F. Sandblom, and J. Sörstedt, "Extended object tracking using a radar resolution model," Aerospace and Electronic Systems, IEEE Transactions on, vol. 48, no. 3, pp. 2371-2386, 2012.

[25] J. Borenstein and L. Ojeda, "Heuristic reduction of gyro drift in gyro-based vehicle tracking," in SPIE Defense, Security, and Sensing. International Society for Optics and Photonics, 2009, pp. $730507-$ 730507.

[26] R. Lamm, B. Psarianos, T. Mailaender et al., Highway design and traffic safety engineering handbook. McGraw-Hill New York, 1999, vol. 2.

[27] R. Bulirsch, "Numerical calculation of the sine, cosine and fresnel integrals," Numerische Mathematik, vol. 9, no. 5, pp. 380-385, 1967.

[28] E. Bertolazzi and M. Frego, "G1 fitting with clothoids," Mathematical Methods in the Applied Sciences, vol. 38, no. 5, pp. 881-897, 2015.

[29] E. D. Dickmanns and A. Zapp, "Autonomous high speed road vehicle guidance by computer vision," in International Federation of Automatic Control. World Congress (10th). Automatic control: world congress., vol. 1,1988
[30] E. Dickmanns and B. Mysliwetz, "Recursive 3-d road and relative egostate recognition," Pattern Analysis and Machine Intelligence, IEEE Transactions on, vol. 14, no. 2, pp. 199-213, Feb 1992.

[31] A. Eidehall, J. Pohl, and F. Gustafsson, "Joint road geometry estimation and vehicle tracking," Control Engineering Practice, vol. 15, no. 12, pp 1484 - 1494, 2007.

[32] D. Schwartz, "Clothoid road geometry unsuitable for sensor fusion clothoid parameter sloshing," in Intelligent Vehicles Symposium, 2003. Proceedings. IEEE, June 2003.

[33] C. Gackstatter, P. Heinemann, S. Thomas, B. Rosenhahn, and G. Klinker, "Fusion of clothoid segments for a more accurate and updated prediction of the road geometry," in Intelligent Transportation Systems (ITSC), 2010 13th International IEEE Conference on, Sept 2010, pp. 16911696.

[34] R. Van der Merwe and E. Wan, "The square-root unscented Kalman filter for state and parameter-estimation," in Acoustics, Speech, and Signal Processing, 2001. Proceedings. (ICASSP '01). 2001 IEEE International Conference on, vol. 6, 2001, pp. 3461-3464 vol.6.

[35] I. Arasaratnam and S. Haykin, "Cubature kalman filters," Automatic Control, IEEE Transactions on, vol. 54, no. 6, pp. 1254-1269, 2009.

[36] A. H. Sayed and T. Kailath, "A state-space approach to adaptive rls filtering," Signal Processing Magazine, IEEE, vol. 11, no. 3, pp. 18-60, 1994.

[37] D. Ballard, "Generalizing the Hough transform to detect arbitrary shapes," Pattern Recognition, vol. 13, no. 2, pp. 111 - 122, 1981.

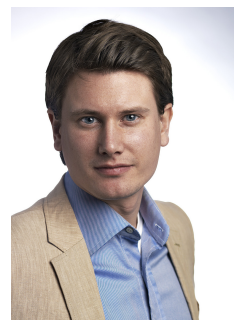

Lars Hammarstrand Lars Hammarstrand was born in Landvetter, Sweden in 1979. He received his M.Sc. and Ph.D. degree in electrical engineering from Chalmers University of Technology, Gothenburg, Sweden, in 2004 and 2010, respectively.

Between 2004 and 2011, he was with the Active Safety and Chassis Department at Volvo Car Corporation, Gothenburg, conducting research on tracking and sensor fusion methods for active safety systems. Currently, Lars is an Assistant Professor at the Signal Processing group at Chalmers University of Technology where his main research interests are in the fields of estimation, sensor fusion, self-localization and mapping, especially with application to self-driving vehicles.

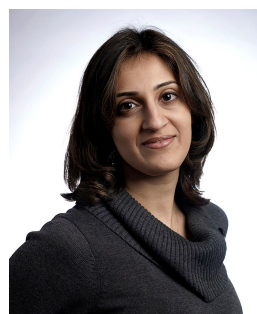

Maryam Fatemi received the B.Sc. degree in electrical engineering from K.N. Toosi University of Technology, Tehran, Iran in 2005, the M.Sc. degree in communication systems engineering from AmirKabir University of Technology, Tehran, Iran in 2008 She is currently working toward her $\mathrm{Ph} . \mathrm{D}$. degree at Chalmers University of Technology. Her research interests include Bayesian Inference and nonlinear filtering with applications to sensor data fusion, autonomous driving and active safety systems

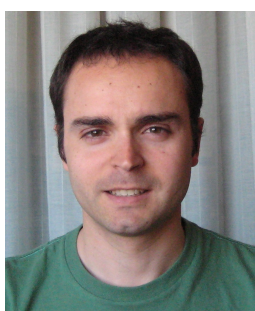

Ángel F. García-Fernández received the telecommunication engineering degree (with honours) and the Ph.D. degree from Universidad Politécnica de Madrid, Madrid, Spain, in 2007 and 2011, respectively.

He is currently a Research Associate in the Department of Electrical and Computer Engineering at Curtin University, Perth, Australia. His main research activities and interests are in the area of Bayesian nonlinear filtering and smoothing.

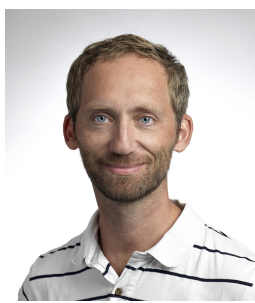

Lennart Svensson was born in Älvängen, Sweden in 1976. He received the M.S. degree in electrical engineering in 1999 and the Ph.D. degree in 2004, both from Chalmers University of Technology, Gothenburg, Sweden.

$\mathrm{He}$ is currently Associate Professor at the Signal Processing group, again at Chalmers University of Technology. His main research interests include Bayesian inference in general, and nonlinear filtering and tracking in particular. 